\title{
DC-derived IL-18 drives Treg differentiation, murine Helicobacter pylori-specific immune tolerance, and asthma protection
}

\author{
Mathias Oertli, ${ }^{1}$ Malin Sundquist, ${ }^{2}$ Iris Hitzler, ${ }^{1}$ Daniela B. Engler, ${ }^{1}$ \\ Isabelle C. Arnold, ${ }^{1}$ Sebastian Reuter, ${ }^{3}$ Joachim Maxeiner, ${ }^{3}$ Malin Hansson, ${ }^{2}$ \\ Christian Taube, ${ }^{3,4}$ Marianne Quiding-Järbrink, ${ }^{2}$ and Anne Müller ${ }^{1}$
}

\begin{abstract}
${ }^{1}$ Institute of Molecular Cancer Research, University of Zürich, Zürich, Switzerland. ${ }^{2}$ Department of Microbiology and Immunology, Sahlgrenska Academy, University of Gothenburg, Gothenburg, Sweden. ${ }^{3}$ III. Medical Clinic, Johannes Gutenberg University, Mainz, Germany. ${ }^{4}$ Department of Pulmonary Medicine, Leiden University Medical Center, Leiden, The Netherlands.
\end{abstract}

\begin{abstract}
Persistent colonization with the gastric bacterial pathogen Helicobacter pylori causes gastritis and predisposes infected individuals to gastric cancer. Conversely, it is also linked to protection from allergic, chronic inflammatory, and autoimmune diseases. We demonstrate here that $H$. pylori inhibits LPS-induced maturation of DCs and reprograms DCs toward a tolerance-promoting phenotype. Our results showed that DCs exposed to $\mathrm{H}$. pylori in vitro or in vivo failed to induce $\mathrm{T}$ cell effector functions. Instead, they efficiently induced expression of the forkhead transcription factor FoxP3, the master regulator of Tregs, in naive T cells. Depletion of DCs in mice infected with $\mathrm{H}$. pylori during the neonatal period was sufficient to break $\mathrm{H}$. pylori-specific tolerance. DC depletion resulted in improved control of the infection but also aggravated $T$ cell-driven immunopathology. Consistent with the mouse data, DCs infiltrating the gastric mucosa of human H. pylori carriers exhibited a semimature DC-SIGN ${ }^{+} \mathrm{HLA}^{-} \mathrm{DR}{ }^{\text {hi }} \mathrm{CD80}^{\mathrm{lo}} \mathrm{CD86}^{\text {lo }}$ phenotype. Mechanistically, the tolerogenic activity of H. pylori-experienced DCs was shown to require IL-18 in vitro and in vivo; DC-derived IL-18 acted directly on $\mathrm{T}$ cells to drive their conversion to Tregs. $\mathrm{CD} 4^{+} \mathrm{CD}^{2} 5^{+}$Tregs from infected wild-type mice but not $\mathrm{Il18}^{-/-}$or Il18 $\mathrm{r}^{-/-}$mice prevented airway inflammation and hyperresponsiveness in an experimental model of asthma. Taken together, our results indicate that tolerogenic reprogramming of DCs ensures the persistence of $H$. pylori and protects against allergic asthma in a process that requires IL-18.
\end{abstract}

\section{Introduction}

DCs are predominantly known for their function as potent inducers of adaptive immunity. However, there is now increasing evidence that certain populations of poorly immunogenic DCs initiate and maintain immunological tolerance through induction of anergy, deletion of autoreactive $\mathrm{T}$ cells, and the instruction and differentiation of Tregs (1). Under steady-state conditions, tissueresident immature DCs express low levels of MHC class II (MHCII), costimulatory molecules, and proinflammatory cytokines but may transform to fully mature DCs upon antigen uptake, accompanied by the concomitant sensing of pathogen-associated molecular patterns (PAMPs) or of "danger" signals released by tissues in distress (2). Such PAMPs or danger-associated molecular patterns (DAMPs) are detected by membrane-bound TLRs or cytoplasmic Nod-like receptors (NLRs) $(3,4)$ and induce the expression of MHCII, CD40, CD80, and CD86 as well as a number of proinflammatory and $\mathrm{T}$ cell-activating cytokines, including IL-1 $\beta$, IL-6, and IL-12 (2). Immature DCs that have taken up antigen, but have not simultaneously been exposed to TLR or NLR ligands, are thought to acquire a semimature state, characterized by high levels of MHCII but low or no expression of costimulatory molecules or proinflammatory cytokines; such semimature $\mathrm{CD} 11 \mathrm{c}^{+} \mathrm{MHCII}{ }^{\text {hi }} \mathrm{C}$ D80 ${ }^{\text {lo }} \mathrm{CD} 6^{\text {lo }} \mathrm{DCs}$ are believed to exhibit tolerogenic (as opposed to stimulatory or immunogenic) properties (1). Tolerogenic DCs function by converting naive $\mathrm{T}$ cells into FoxP $3^{+}$Tregs with sup-

Conflict of interest: The authors have declared that no conflict of interest exists. Citation for this article: J Clin Invest. 2012;122(3):1082-1096. doi:10.1172/JCI61029. pressive activity; Treg induction is achieved through antigen presentation in the absence of costimulatory signals or cytokines, either alone or in combination with the production of soluble and membrane-bound tolerogenic factors, such as IL-10, TGF- $\beta$, retinoic acid, and programmed death ligands $(1,5)$.

Persistent infection with the gram-negative gastric bacterial pathogen Helicobacter pylori results in chronic gastritis (6) and predisposes carriers to a high risk of developing gastric and duodenal ulcers, gastric cancer, and gastric mucosa-associated lymphoid tissue lymphoma (7-9). We and others have shown previously that MHCII-restricted T cells are required for the control of this extracellular pathogen under conditions of experimental infection in naive mice (10) and for the development of vaccineinduced protective immunity $(11,12)$. Th1-polarized, pathogenic $\mathrm{CD}^{+} \mathrm{T}$ cells further represent the driving force behind the infection-associated gastric preneoplastic immunopathology that manifests histologically as atrophic gastritis, epithelial hyperplasia, and intestinal metaplasia in infected rodents and in a subset of chronically infected humans $(10,13-15)$. Consequently, targeting $\mathrm{T}$ cells pharmacologically prevents and even reverses the gastric immunopathology associated with chronic Helicobacter infection $(16,17)$. Interestingly, the outcome of the Helicobacter/host interaction varies dramatically depending on the age at the time of infection. Whereas mice infected as fully immunocompetent adults develop preneoplastic lesions within 2 to 4 months of infection with virulent Helicobacter strains, mice infected during the neonatal period are protected against gastric immunopathology due to their development of immune tolerance to the pathogen (18). 
The depletion of Tregs breaks neonatally acquired tolerance and results in a dramatic reduction of bacterial loads and the development of Th1-associated immunopathology (18). Tregs induced during neonatal infection are further both required and sufficient to mediate the $H$. pylori-induced, but antigen-independent, crossprotection against allergic asthma that is evident in human carriers of $H$. pylori (19-22) and in experimentally infected mice (23). In an ovalbumin sensitization/challenge model of allergic asthma, neonatally infected mice were completely protected against the clinical and histopathological features of asthma, i.e., airway hyperresponsiveness, tissue inflammation, and bronchoalveolar eosinophilia (23). Depletion of Tregs abrogated protection, and the adoptive transfer of Tregs was sufficient to transfer protection against ovalbumin-induced asthma from neonatally infected donors to naive recipients (23).

Here we show that $H$. pylori possesses the ability to profoundly impact the DC maturation process and to convert immature DCs to tolerogenic DCs in vitro and in vivo. H. pylori-tolerized DCs are incapable of activating effector functions in naive $\mathrm{T}$ cells but become very efficient inducers of FoxP $3^{+}$Tregs. DC depletion breaks neonatally induced, $H$. pylori-specific tolerance. We further show that large numbers of semimature DCs are detectable in the chronically infected gastric mucosa of human $H$. pylori carriers and that the tolerogenic properties of $H$. pylori-experienced DCs require the secretion of IL-18 and its direct action on naive T cells. Taken together, our results suggest that $H$. pylori skews the host's immune response toward tolerance over immunity through its direct effects on DCs and that the H. pylori/DC interaction forms the mechanistic basis for bacterial persistence and for the protection against asthma that is a hallmark of $H$. pylori-infected individuals.

\section{Results}

H. pylori infection prevents DC maturation in a contact-dependent manner. To assess the effects of $H$. pylori infection on DC maturation, we generated immature bone marrow-derived DCs (BM-DCs) and treated the cells with E. coli LPS to induce their maturation, either in the absence or presence of $H$. pylori. Flow cytometric analysis of CD80, CD86, and CD40 expression revealed that LPS treatment efficiently induced DC maturation, which was strongly inhibited by the presence of live H. pylori (Figure 1, A and B). The infection alone had no effect on the maturation state of DCs (Figure 1, A and $\mathrm{B}$ ). The expression of DC maturation markers was paralleled by secretion of IL-12 and IL- 6 upon LPS treatment, which was also inhibited by $H$. pylori (Figure 1C). Expression of the antiinflammatory cytokine IL-10 was inversely correlated with the other cytokines; its expression was increased by the infection but was not elevated in the LPS-treated samples (Figure 1C). The inhibition of DC maturation did not depend on the presence of a functional cytotoxin-associated gene ( $\mathrm{Cag}$ ) pathogenicity island-encoded type IV secretion system, as an $H$. pylori mutant deficient for an essential component of the system, CagE, was as efficient as wild-type bacteria in preventing LPS-induced DC maturation, as assessed by staining for CD80 (Figure 1D) and by ELISA for IL-12p40 (Figure 1E). In contrast, direct contact between wild-type bacteria and the cells was required for this effect, as a transwell filter that prevented attachment of the bacteria also impaired the inhibition of LPS-induced DC maturation (Figure 1, F and G). The observed consequences of $H$. pylori infection were not due to cytotoxic effects of the bacteria on cultured DCs and were at least partially phenocopied by treatment with H. pylori extract (Supple- mental Figure 1, A-C; supplemental material available online with this article; doi:10.1172/JCI61029DS1). We further alternatively used the TLR2 and TLR9 ligands PAM3Cys and CPG to induce DC maturation; H. pylori infection also efficiently prevented CD80 expression and IL-12 secretion induced by these stimuli (Figure 1, $\mathrm{H}$ and $\mathrm{I}$, and data not shown). Interestingly, we could not attribute the effects of $H$. pylori on DCs to the C-type lectin receptor DC-specific ICAM3-grabbing nonintegrin (DC-SIGN), which was previously demonstrated to mediate immune escape by $H$. pylori (24). DCs transgenically expressing human DC-SIGN (hDC-SIGN) under the control of the $c d 11 c$ promoter did not differ from wildtype DCs, with respect to the inhibitory effects of H. pylori on LPSinduced DC maturation; mice expressing hDC-SIGN (25) further did not differ from wild-type littermates in terms of $H$. pylori colonization levels (Supplemental Figure 1, D-F). Taken together, the results indicate that $H$. pylori has evolved a mechanism of impairing DC function in a manner that depends on direct contact but is independent of the type IV secretion system.

H. pylori exposure generates tolerogenic DCs with the ability to convert naive T cells to FoxP $3^{+}$Tregs in a contact-and TGF- $\beta$-dependent manner. One of the inherent characteristics of semimature DCs is their ability to convert naive $\mathrm{T}$ cells into $\mathrm{FoxP}^{+}$Tregs with suppressive activity. To assess whether the exposure to $H$. pylori induces such tolerogenic DCs, we compared the ability of untreated and $H$. pyloriinfected BM-DCs to convert naive $\mathrm{T}$ cells to FoxP3 ${ }^{+}$Tregs (Figure 2A). In line with their semimature phenotype ( $\mathrm{MHCII}{ }^{\text {hi }} \mathrm{CD} 80^{\text {lo }}$ $\mathrm{CD} 86^{\text {lo }} \mathrm{CD} 40^{\text {lo }}$; Figure $1, \mathrm{~A}$ and $\mathrm{B}$; data not shown for MHCII), BM-DCs that had been infected with $H$. pylori induced FoxP3 ${ }^{+}$ Tregs significantly more efficiently than naive BM-DCs, when cultured in the presence of anti-CD3 cross-linking antibody and TGF- $\beta$ (Figure 2, A and B). Similarly, H. pylori-infected BM-DCs loaded with ovalbumin were better able than their naive counterparts to trigger the conversion of ovalbumin-specific (OTII) T cells to FoxP3 ${ }^{+}$Tregs (Figure 2, C and D). Direct contact between the infected BM-DCs and T cells was required for their efficient conversion to FoxP3 $3^{+}$Tregs, as the separation of both cell populations by a transwell filter abrogated the effect (Figure 2E). Interestingly, DCs isolated from the bone marrow of gene-targeted mice lacking MyD88 or TLR2, an important pattern recognition receptor for $H$. pylori $(26,27)$, were as capable of Treg conversion upon $H$. pylori exposure as wild-type BM-DCs (Figure 2F). We next immunomagnetically purified CD $11 \mathrm{c}^{+}$DCs from the mesenteric lymph nodes (MLNs) of C57BL/6 mice to more than $80 \%$ purity and infected them with live H. pylori prior to coculture with naive T cells. Infected MLN-derived DCs (MLN-DCs) were significantly more potent inducers of FoxP3 expression in T cells than naive MLN-DCs in the presence of anti-CD3 cross-linking antibody (Figure 2, G and $\mathrm{H}$ ) or upon ovalbumin-specific stimulation of OTII T cells (Figure 2, I and J), indicating that the tolerizing effects of $H$. pylori exposure are common to both BM-DC and MLN-DC populations.

$H$. pylori-experienced DCs are incapable of activating $T$ cell effector responses. To examine whether the exposure of DCs to $H$. pylori not only renders them tolerogenic, but at the same time impairs their ability to activate $T$ cell effector functions and to induce Th1 differentiation, we cocultured $H$. pylori-infected BM-DCs with naive OTII T cells under conditions of anti-CD3 cross-linking or ovalbumin-specific priming. Infected BM-DCs were significantly less able than naive BM-DCs to induce IFN- $\gamma$ expression and secretion in T cells activated by CD3 cross-linking or ovalbumin priming, as determined by intracellular cytokine staining and ELISA 
A

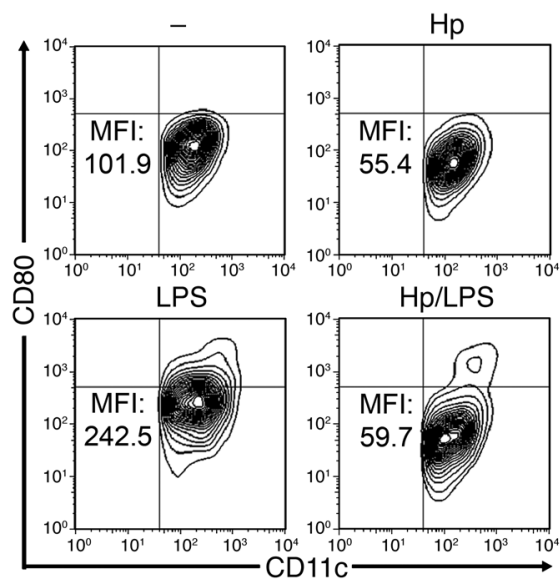

B

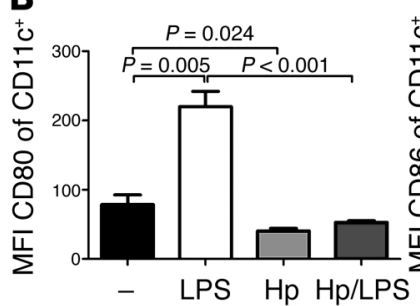

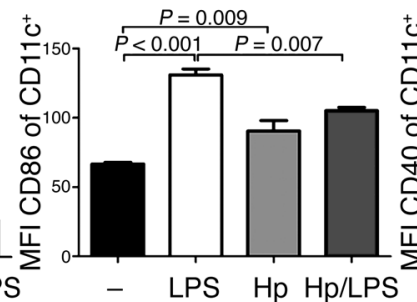

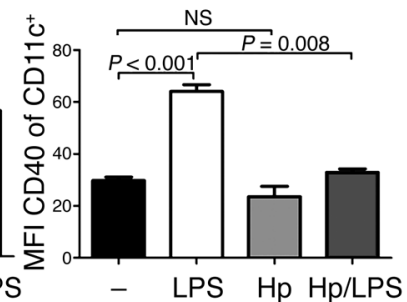

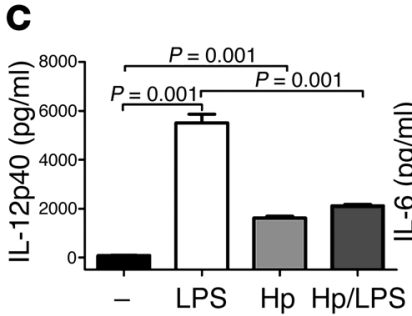

- LPS Hp Hp/LPS

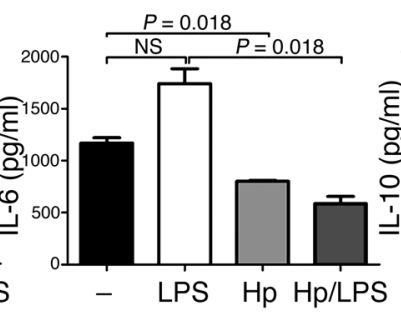

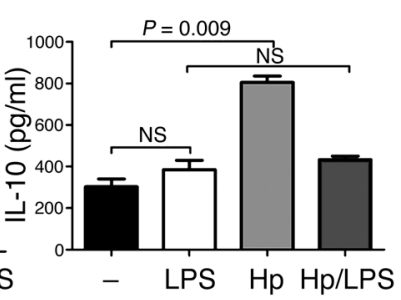

G
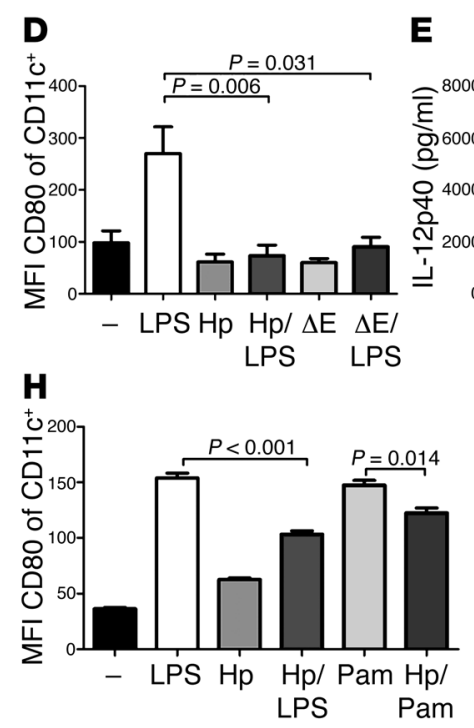

E

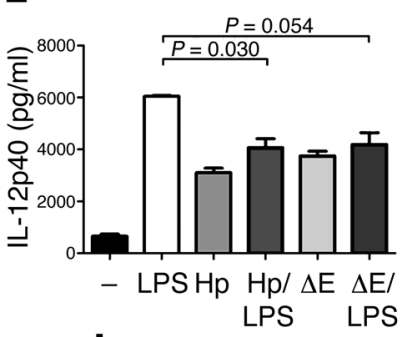

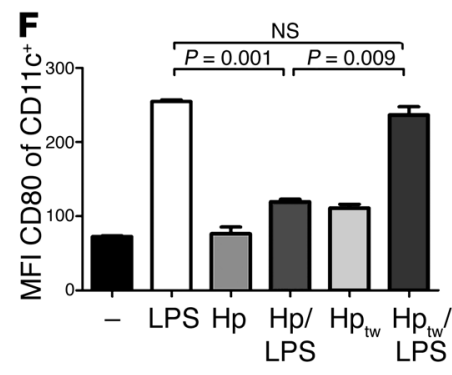

LPS

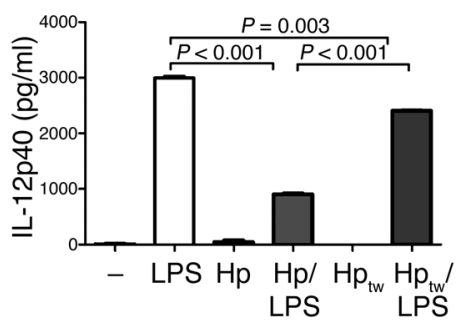

I

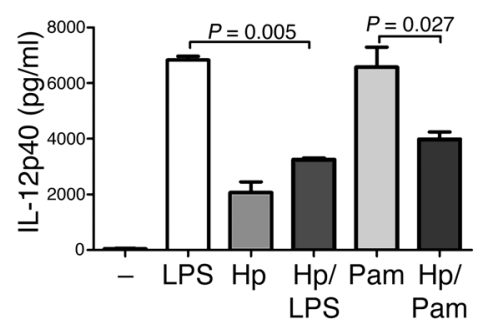

Figure 1

The TLR ligand-induced maturation of DCs is impaired by $H$. pylori infection. (A-C) BM-DCs were infected with $H$. pylori (Hp) strain PMSS1 at a MOI of 50 and/or treated with $0.5 \mu \mathrm{g} / \mathrm{ml} \mathrm{E}$. coli LPS for 16 hours prior to (A and $\mathbf{B}$ ) the flow cytometric analysis of CD80, CD86, and CD40 expression and (C) the quantification of IL-12p40, IL-6, and IL-10 secretion by ELISA. Representative FACS plots are shown for CD80 in A, and the average MFI of CD80, CD86, and CD40 expression of all CD11 $\mathrm{c}^{+}$cells is shown in B. (D and E) BM-DCs were infected with $H$. pylori strain PMSS1 or its isogenic mutant, PMSS1 $\triangle \mathrm{CagE}(\Delta \mathrm{E})$, and/or treated with LPS for 16 hours and (D) assessed for CD80 expression and (E) IL-12p40 secretion. (F and G) BM-DCs were infected with H. pylori strain PMSS1 and/or treated with LPS for 16 hours and $(\mathbf{F})$ assessed for CD80 expression and (G) IL-12p40 secretion; bacteria were separated from the cells by a transwell (tw) filter where indicated. (H and I) BM-DCs were infected with H. pylori strain PMSS1 and/or treated with $0.5 \mu \mathrm{g} / \mathrm{ml} \mathrm{LPS} \mathrm{or} 5 \mu \mathrm{g} / \mathrm{ml} \mathrm{Pam3Cys} \mathrm{(Pam)} \mathrm{for} 16$ hours and assessed for (H) CD80 expression and (I) IL-12p40 secretion. Data are representative of (D-I) at least 3 and (A-C) up to 8 independent experiments and are represented as mean \pm SEM of triplicate cultures. $P$ values were calculated using Student's $t$ test.

(Figure 3, A and B). T cell expansion, as assessed by $\left[{ }^{3} \mathrm{H}\right]$ thymidine incorporation, was impaired as well (Figure 3C). To confirm these results with MLN-DCs, immunomagnetically isolated CD $11 c^{+}$DCs from the MLNs of naive mice were infected ex vivo and cocultured with OTII-transgenic T cells under conditions of CD3 cross-linking or ovalbumin-specific priming. As observed with the BM-DCs, infected MLN-DCs were significantly less capable of inducing IFN- $\gamma$ production upon CD 3 cross-linking or ovalbumin-specific priming than uninfected MLN-DCs (Figure 3, D-F). The combined results suggest that $H$. pylori-experienced DCs are better inducers of Tregs, but worse inducers of effector $T$ cells, than naive DCs without prior exposure to the bacteria.
H. pylori-experienced DCs fail to induce asthma in a DC-mediated ovalbumin sensitization and challenge model. We and others have shown recently that infection with $H$. pylori protects against allergic asthma in humans (19-22) and in mouse models of the disease (23). A hallmark of infected mice protected against asthma was their pulmonary infiltration with immature, $\mathrm{CD} 11 \mathrm{c}^{+} \mathrm{MHCII}{ }^{\mathrm{lo}} \mathrm{DCs}$, which were much less abundant in asthmatic mice (23). To determine whether exposure to $H$. pylori indeed generates DCs that are unable to promote asthma, we established a model in which 1 intranasal dose of ovalbumin-loaded DCs was used to sensitize mice to ovalbumin, followed by challenge with aerosolized ovalbumin 2 weeks later. Uninfected, ovalbumin-loaded DCs were compared 

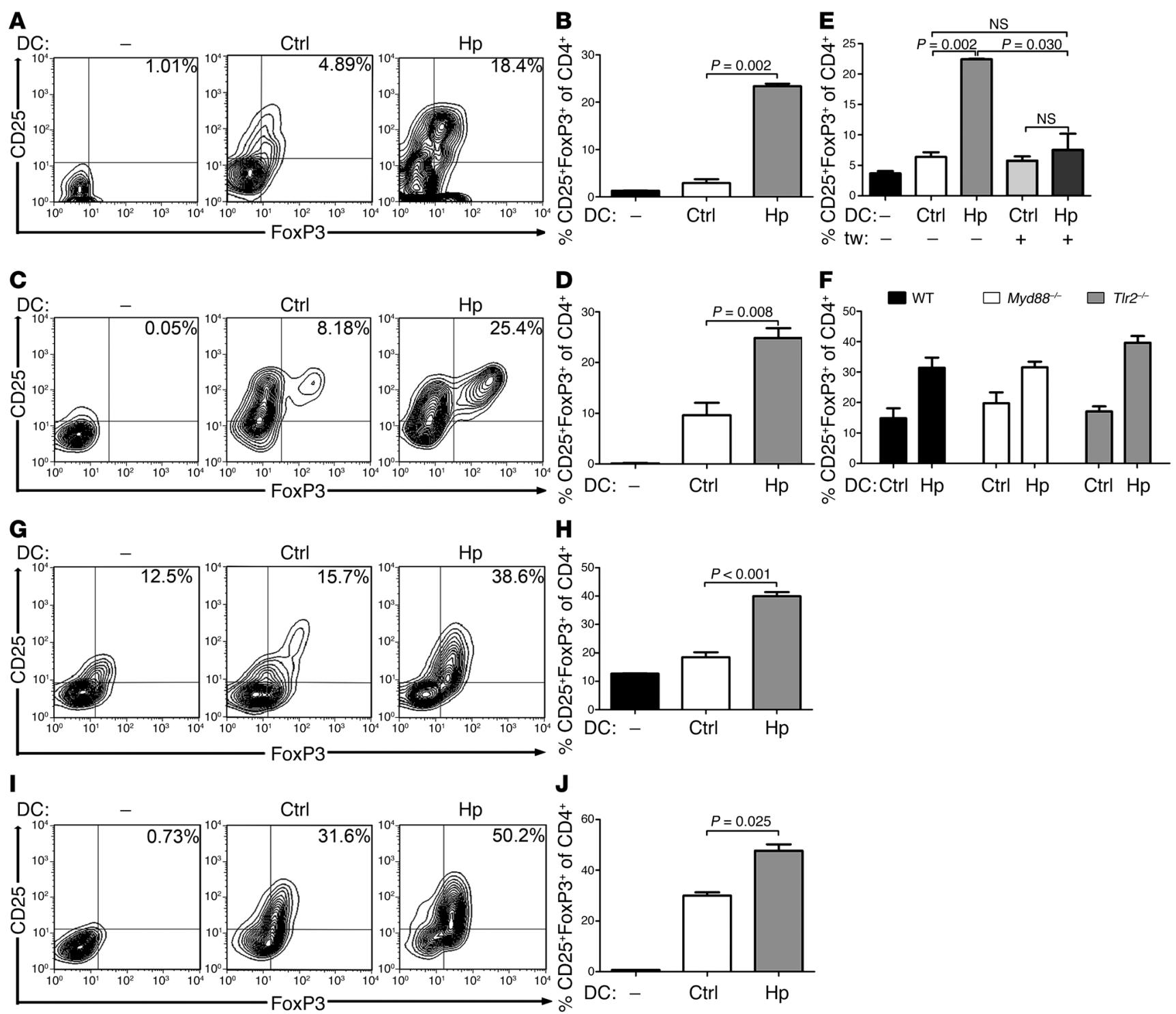

Figure 2

H. pylori-experienced DCs induce FoxP3 expression in naive T cells. (A and B) BM-DCs were infected with H. pylori PMSS1 (MOI 50); after 16 hours, bacteria were killed with antibiotics. DCs were cocultured with immunomagnetically isolated, splenic CD4+CD25- T cells for 3 days in the presence of rTGF- $\beta$, rlL-2, and anti-CD3e mAb prior to flow cytometric analysis of CD4, CD25, and FoxP3 expression. (A) Representative plots of the $\mathrm{CD}^{+}$gate, $(\mathbf{B})$ along with mean \pm SEM of triplicate cocultures. T cells cultured in the absence of DCs served as controls (ctrls). (C and $\left.\mathbf{D}\right)$ BM-DCs were treated as described in A and B and were additionally loaded with $20 \mu \mathrm{g} / \mathrm{ml}$ ovalbumin prior to coculturing with CD4+CD25- OTII T cells in the presence of rTGF- $\beta$ and rlL-2. (C) Representative CD25 and FoxP3 plots of the CD4+ gate, (D) along with mean \pm SEM of triplicates. (E) BM-DCs and T cells were treated as described in A, except that both populations were separated by a transwell filter where indicated. (F)

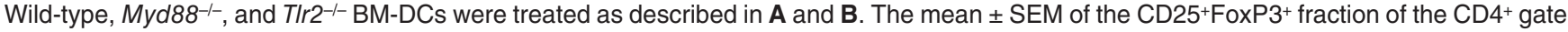
of triplicate cocultures is shown in $\mathbf{E}$ and $\mathbf{F}$. (G and $\mathbf{H})$ Immunomagnetically isolated, MLN-derived CD11 $\mathrm{C}^{+}$DCs were treated and cocultured with T cells as described in A and $\mathbf{B}$. (I and $\mathbf{J})$ MLN-derived CD11 $\mathbf{c}^{+}$DCs were treated and cocultured with OTII T cells as described in $\mathbf{C}$ and $\mathbf{D}$. ( $\mathbf{G}$ and $\mathbf{I})$ Representative FACS plots are shown, $(\mathbf{H}$ and $\mathbf{J})$ along with mean \pm SEM of triplicate cocultures. Numbers indicate the percentage of FoxP $3+C D 25+$ cells. Data are representative of at least 3 and up to 8 experiments.

with $H$. pylori-infected, ovalbumin-loaded DCs, which had been subjected to antibiotic therapy to kill the bacteria prior to intranasal administration. Additional control groups received DCs that had not been pulsed with antigen. Mice that had received ovalbumin-loaded DCs developed characteristic airway hyperresponsiveness, as assessed upon methacholine inhalation (Figure 4, A and B), and exhibited high levels of peribronchiolar and perivascular inflammation and extensive goblet cell metaplasia (Figure 4, C-E). They further showed high levels of bronchoalveolar infiltration, as assessed by quantification of total cells in the bronchoalveolar lavage fluid (BALF) (Figure 4F). Whereas the BALF from nonasthmatic mice predominantly contained alveolar macrophages, the fraction of macrophages decreased in the BALF of asthmatic recipients of ovalbumin-loaded DCs (Figure 4G). The predomi- 

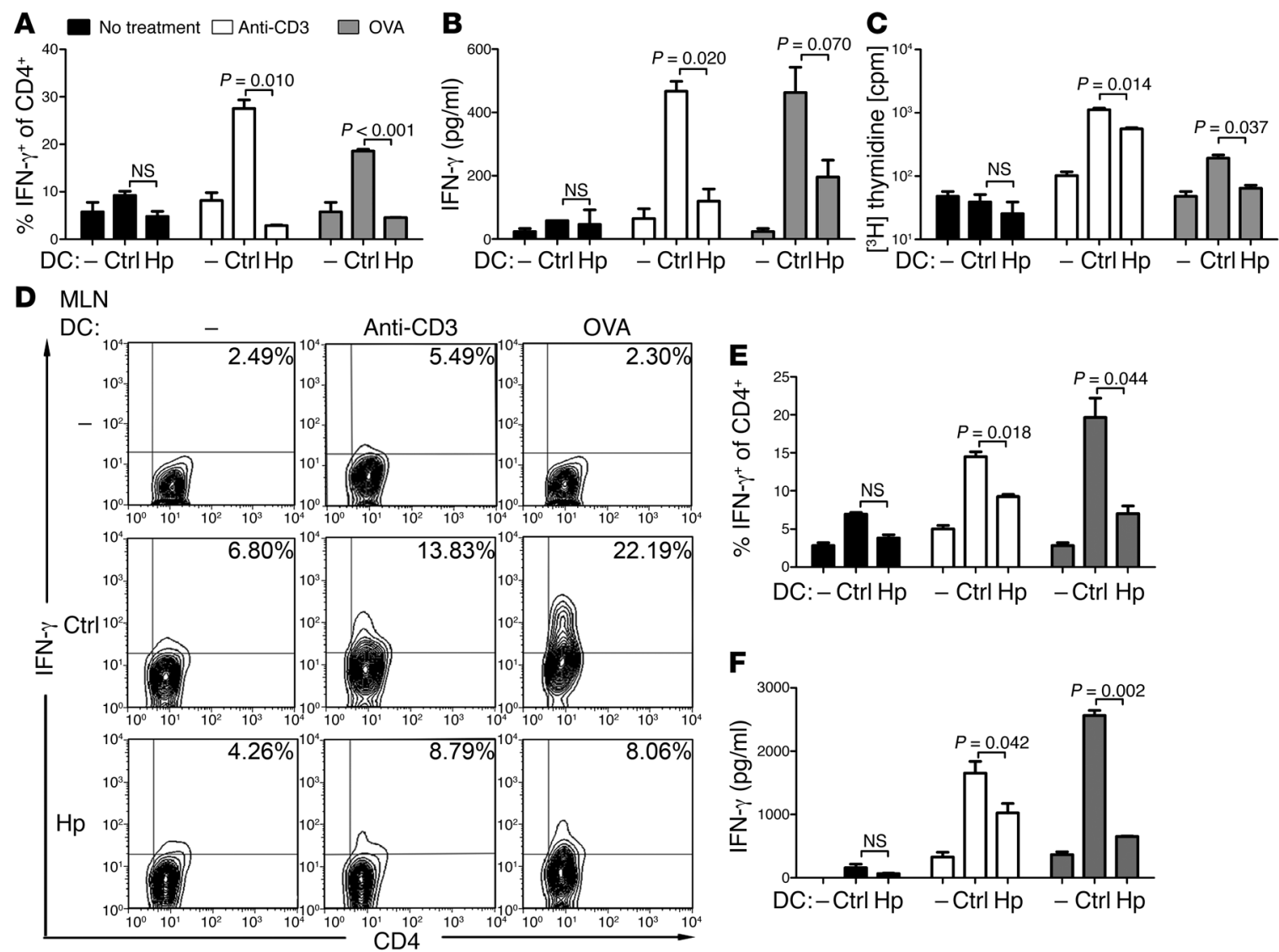

Figure 3

H. pylori infection impairs the ability of DCs to activate T cell effector functions. (A-C) BM-DCs were infected as described in Figure 2, A and B, and/or loaded with $20 \mu \mathrm{g} / \mathrm{ml}$ ovalbumin prior to coculturing with immunomagnetically isolated, splenic OTII CD4+CD25- T cells for 3 days in

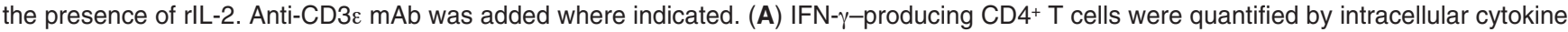
staining, and (B) IFN- $\gamma$ secretion into the supernatant was measured by ELISA. (C) Proliferation of parallel cocultures was determined by $\left[{ }^{3} \mathrm{H}\right]$ thymidine incorporation. T cells cultured without DCs served as controls $(-)$. (D-F) Immunomagnetically isolated, MLN-derived CD11C ${ }^{+}$ DCs were infected and/or loaded with $20 \mu \mathrm{g} / \mathrm{ml}$ ovalbumin prior to coculturing with CD4+CD25- T cells in the presence of rlL-2 and anti-CD3 $\varepsilon$ mAb. (D) Representative FACS plots demonstrating intracellular IFN- $\gamma$ are shown, (E) along with mean \pm SEM of triplicate cocultures and (F) IFN- $\gamma$ secretion into the supernatant as determined by ELISA. Numbers indicate the percentage of IFN- $\gamma^{+}$cells of the CD4+ gate. All data are representative of at least 3 independent experiments.

nant populations of infiltrating cells in asthmatic mice were lymphocytes, neutrophils, and eosinophils (Figure 4, H-J). None of the described symptoms of asthma were elicited by ovalbumin-loaded DCs that had previously been exposed to H. pylori or by DCs not loaded with allergen (Figure 4). In conclusion, the ovalbumin/DCinduced asthma model confirms the inability of infected DCs to prime antigen-specific effector $\mathrm{T}$ cell responses.

Neonatally induced $H$. pylori-specific immune tolerance requires DCs. Hypothesizing that $H$. pylori infection of mice should generate tolerogenic rather than immunogenic DCs in light of our in vitro findings, we infected mice with $H$. pylori PMSS1, a virulent patient isolate that harbors a functional Cag pathogenicity island, establishes persistent infection in mice, and induces the full range of gastric inflammation and preneoplastic pathology that characterizes the $H$. pylori-infected gastric mucosa of susceptible, chronically infected patients (18). In addition to mice infected at 6 weeks of age (i.e., as adults), we included mice that had been infected with H. pylori PMSS1 during the neonatal period (i.e., at 7 days of age). Neonatally infected mice develop tolerance rather than immunity to H. pylori infection (18); the neonatal infection model is therefore of particular interest in the context of elucidating the tolerogenic properties of $H$. pylori-infected DCs. As the MLNs represent 1 out of 2 documented sites of $H$. pylori-specific $\mathrm{T}$ cell priming alongside the Peyer's patches $(16,17,28,29)$, we immunomagnetically isolated MLN-DCs from individual mice from uninfected, neonatally infected, and adult-infected groups and compared them with respect to their ability to induce FoxP3 expression in naive T cells. MLN-DCs from infected mice were significantly more capable of inducing FoxP3 expression in T cells than those isolated from uninfected controls (Figure 5, A and B). The tolerogenic, FoxP3inducing properties were particularly evident in DCs from neonatally infected, tolerant mice (Figure 5, A and B), which are colonized more densely than their adult-infected counterparts (Figure 5C) due to their lack of $\mathrm{CD}^{+} \mathrm{T}$ cell infiltration into the gastric mucosa (Figure 5D) and their failure to generate Th1 responses to the infection (18). Indeed, DCs from neonatally infected mice were less capable of inducing Th1 effector responses in naive $\mathrm{T}$ cells than DCs from mice infected as adults (Figure 5E), underscoring the 


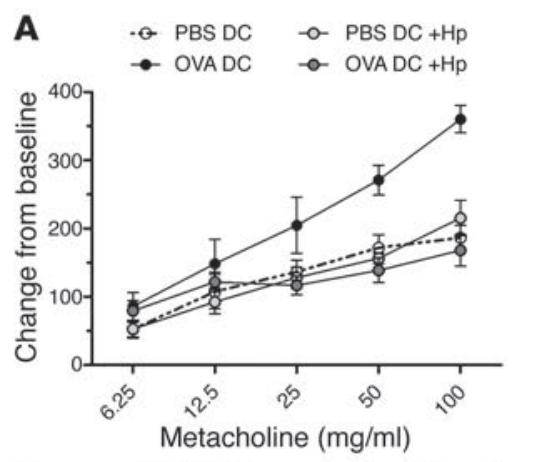

D
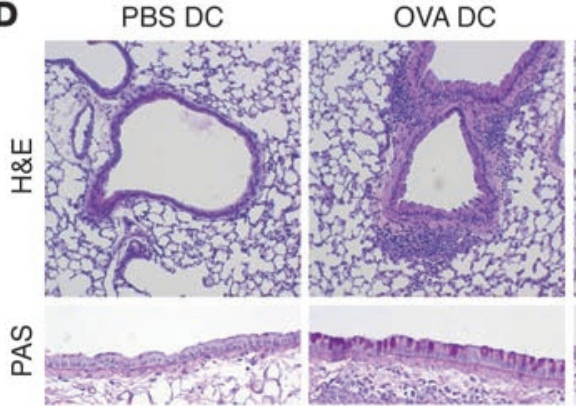

$\mathbf{F}$
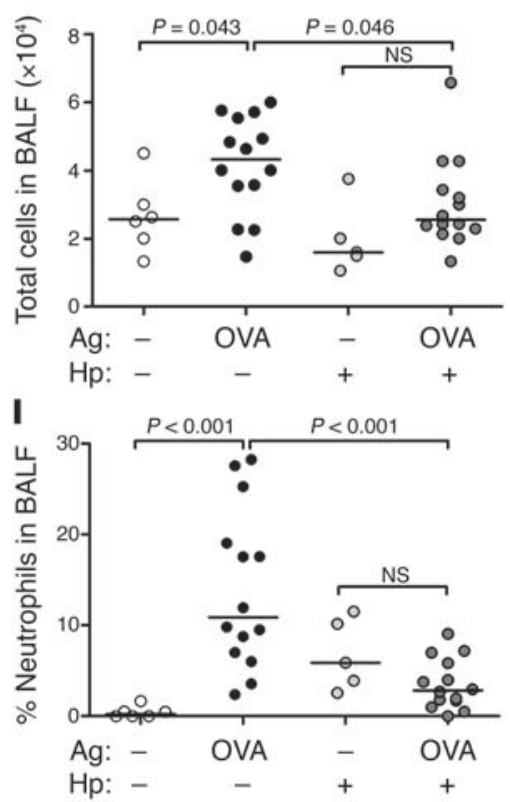

B

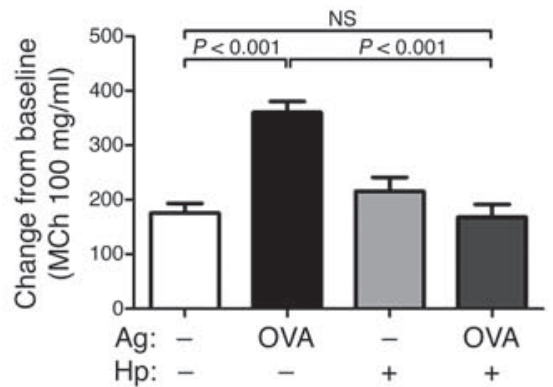

PBS DC Hp
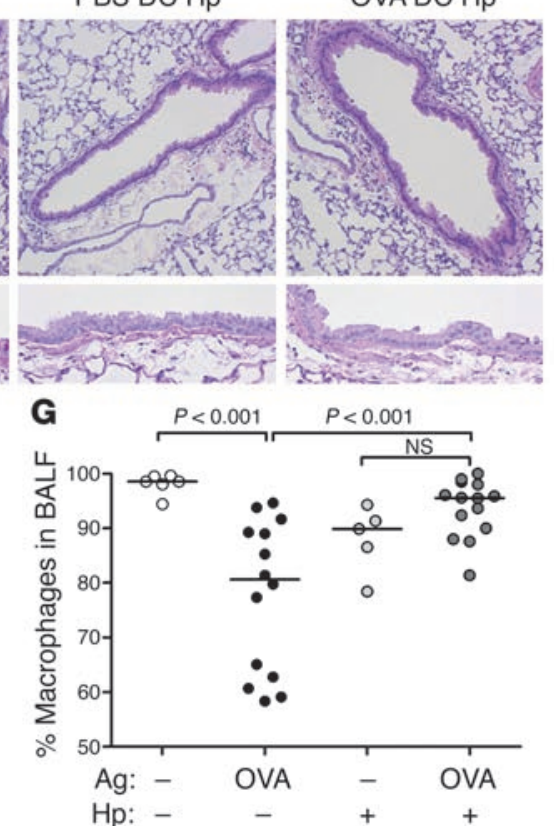

J

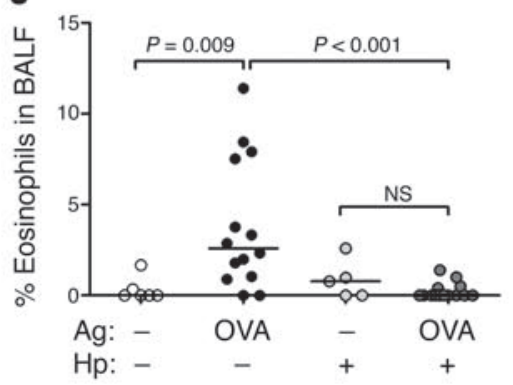

C

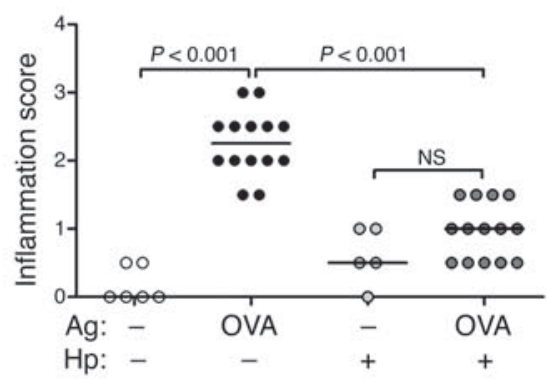

E

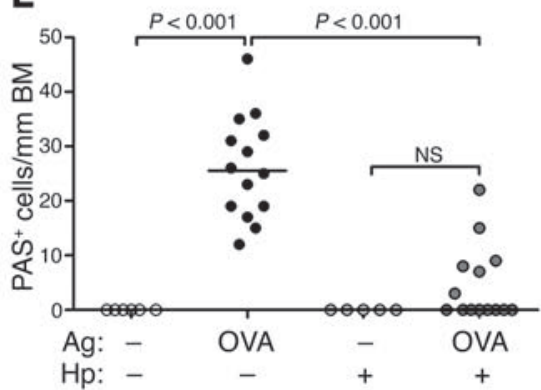

H

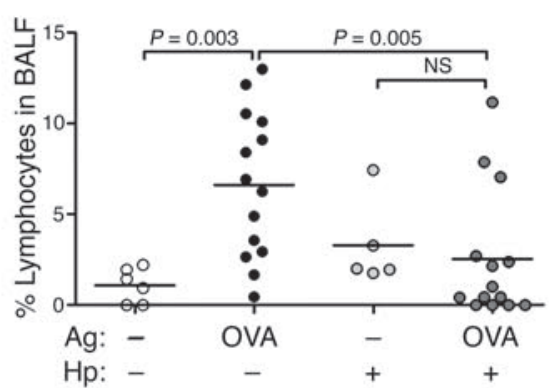

Figure 4

H. pylori-experienced DCs fail to induce asthma-like symptoms in an adoptive transfer model of DC-driven airway hyperresponsiveness. BM-DCs were infected and treated as described in Figure 2, A and B, and/or loaded with either $20 \mu \mathrm{g} / \mathrm{ml}$ ovalbumin (OVA-DC \pm Hp [either infected or not infected with $H$. pylori]) or PBS only (PBS-DC $\pm \mathrm{Hp}$ ). $1 \times 10^{6} \mathrm{BM}-\mathrm{DC}$ per mouse were administered intranasally for the purpose of ovalbumin-specific sensitization; all mice were challenged 2 weeks later with aerosolized ovalbumin and assessed for the development of airway hyperresponsiveness and tissue inflammation. ( $\mathbf{A}$ and $\mathbf{B}$ ) Airway hyperresponsiveness, $(\mathbf{A})$ as assessed by challenge with increasing doses of methacholine and (B) the highest dose of $100 \mathrm{mg} / \mathrm{ml}$, respectively. (C-E) Tissue inflammation and goblet cell metaplasia, as assessed on H\&Eand PAS-stained tissue sections. Representative micrographs are shown in D. Original magnification, $\times 100$ (H\&E); $\times 400$ (PAS). Inflammation and PAS scores are shown in $\mathbf{C}$ and E. (F) Total cells contained in $1 \mathrm{ml}$ BALF. Percentages of (G) macrophages, (H) lymphocytes, (I) neutrophils, and $(\mathbf{J})$ eosinophils in $1 \mathrm{ml} \mathrm{BALF}$, as determined by differential staining. In the scatter plots shown in $\mathbf{C}, \mathbf{E}$, and $\mathbf{F}-\mathbf{J}$, each data point represents an individual mouse. Data are representative of 3 independent experiments. Horizontal lines represent the median. 
A

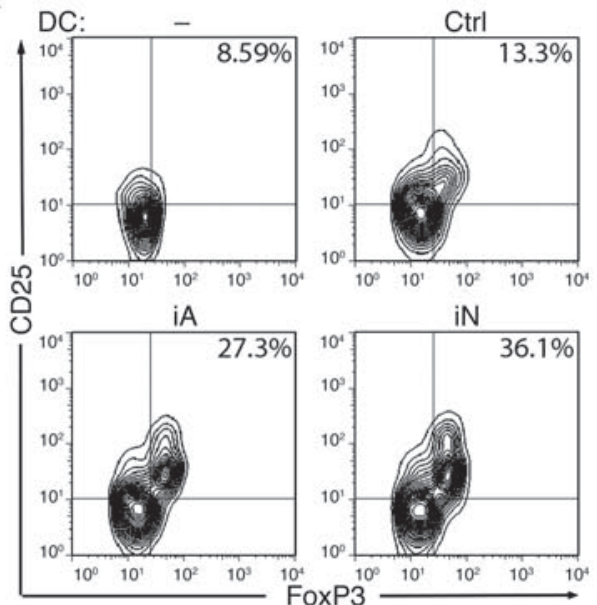

F

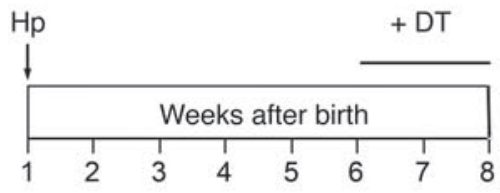

I

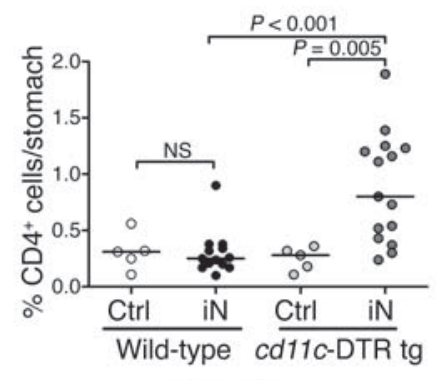

B
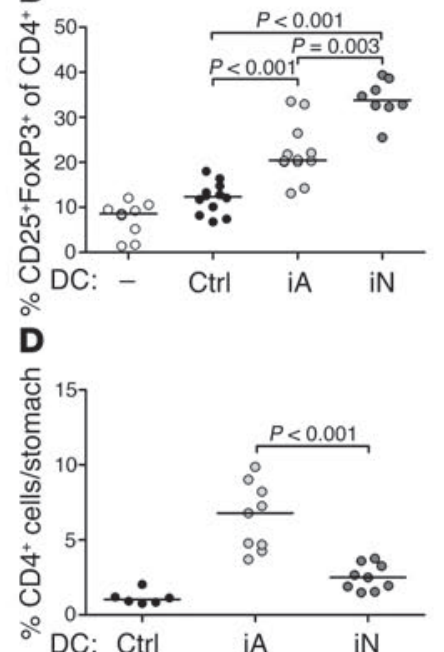

G

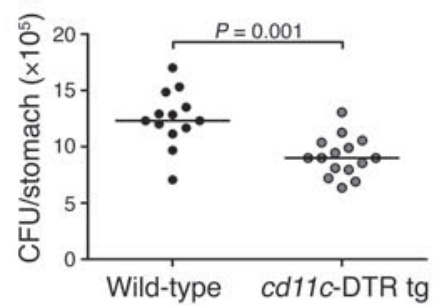

J

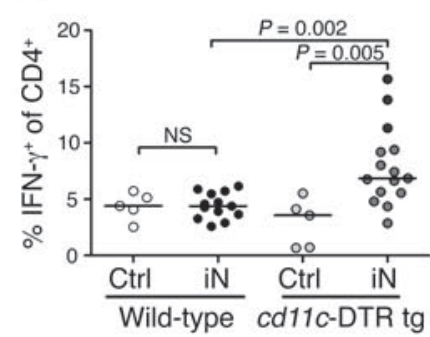

C

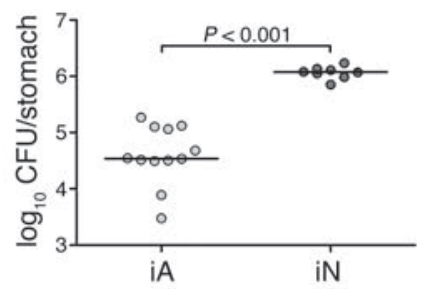

E

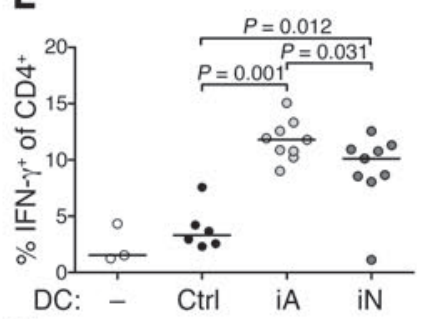

H

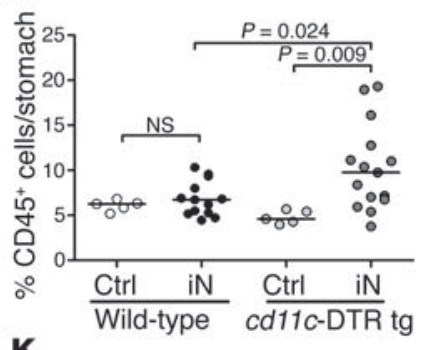

K

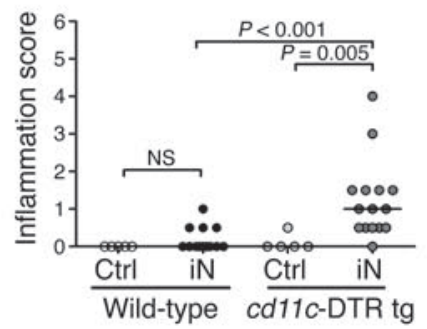

L

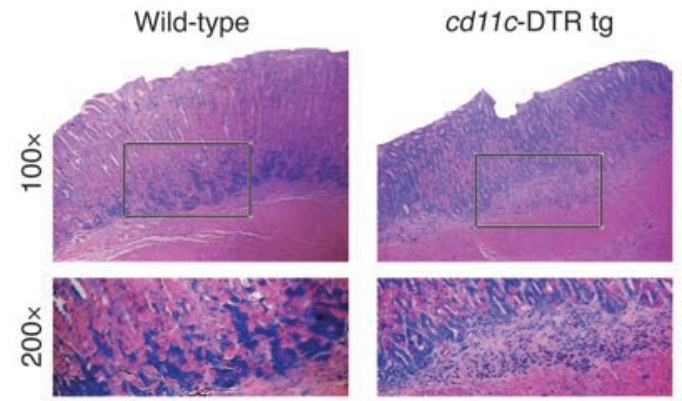

Figure 5

H. pylori-exposed DCs exhibit tolerogenic properties and are required for tolerance in vivo. (A-E) C57BL/6 mice were infected with $H$. pylori at 7 days (iN) or 6 weeks (iA) of age. Upon sacrifice, CD11 $\mathrm{C}^{+}$MLN-DCs were immunomagnetically isolated; cocultured for 3 days with splenic CD4+CD25- T cells, rTGF- $\beta$, rlL-2, and anti-CD3ع mAb; and subjected to flow cytometric analysis of CD4, CD25, and FoxP3 expression. (A) $\mathrm{CD} 25$ and FoxP3 staining of the CD4+ gate is shown for representative donors and (B) quantified for all donors. Numbers indicate the percentage of FoxP3 $3^{+}$CD25+ cells. (C) H. pylori colonization of mice analyzed in B. (D) $C D 4^{+} \mathrm{T}$ cell infiltration into the gastric mucosa of mice shown in B and C. (E) DCs prepared, as described in A, were cultured with CD4+CD25- T cells, rlL-2, and anti-CD3\& mAb and subjected to intracellular IFN- $\gamma$ staining. Each symbol represents an individual donor, and data are pooled from 2 experiments in B-E. (F-L) cd11c-DTR tg mice and their wild-type littermates were infected at 1 week of age with $H$. pylori strain PMSS1 or remained uninfected. (F) All mice received diphtheria toxin during the final 2 weeks of the experiment and were sacrificed 8 weeks after infection. (G) Gastric H. pylori colonization. (H and I) Gastric CD45+ leukocyte and $\mathbf{C D} 4^{+} T$ cell infiltration. (J) Intracellular IFN- $\gamma$ expression by gastric CD4 ${ }^{+} \mathrm{T}$ cells. ( $\mathbf{K}$ and $\mathbf{L}$ ) Gastric histopathology, as assessed on Giemsa-stained sections. Representative micrographs are shown in L. Original magnification, $\times 100$ (top); $\times 200$ (bottom). Inflammation scores are shown in $\mathbf{K}$. Data in $\mathbf{F}-\mathbf{L}$ are pooled from 3 experiments. Horizontal lines indicate the medians. 
A
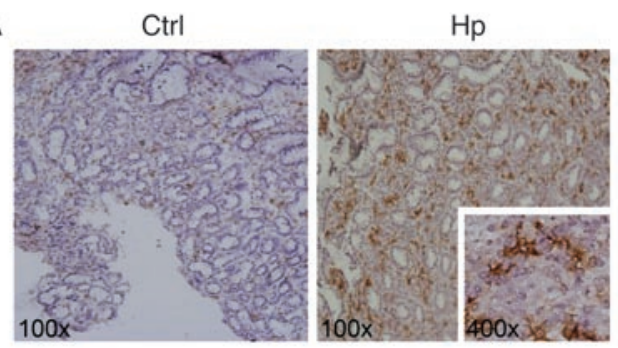

C
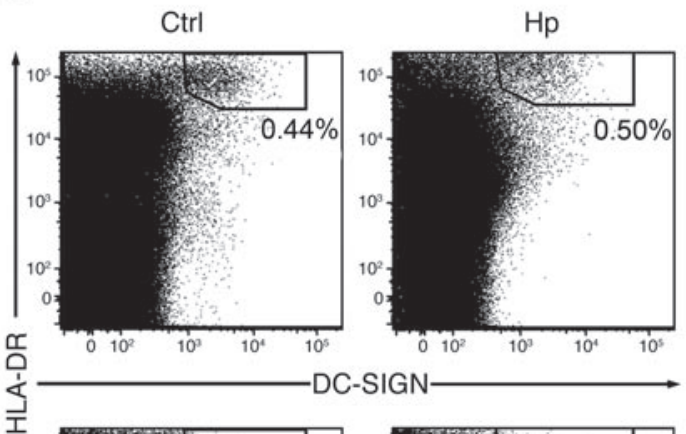

-SIGN
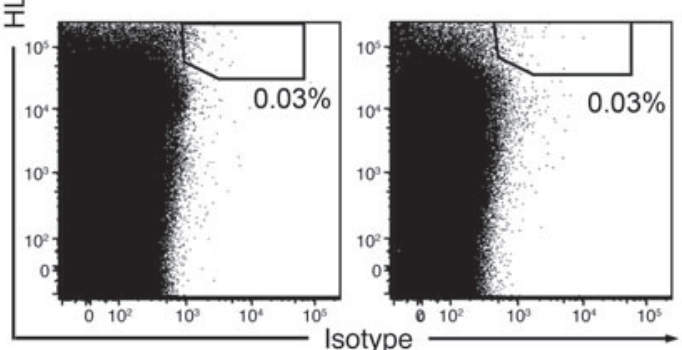

B

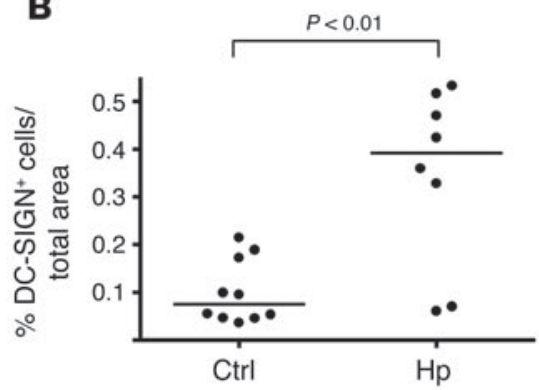

D

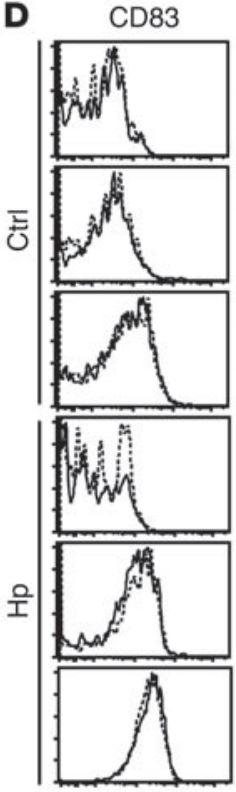

CD80

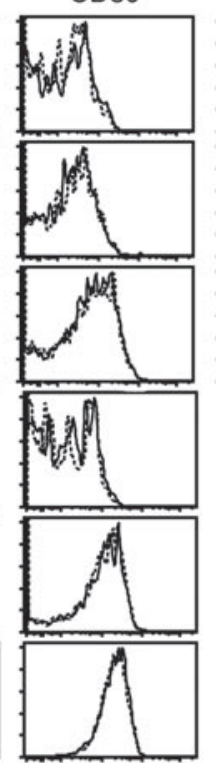

CD86

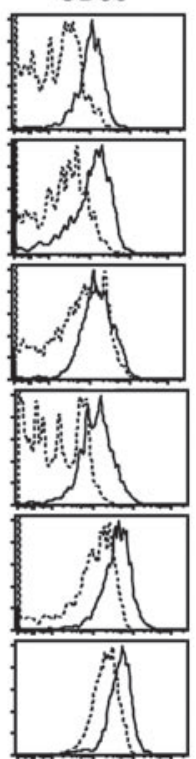

Figure 6

Human gastric DCs exhibit a semimature phenotype in infected patients. (A and B) Antral biopsies were collected from healthy uninfected and $H$. pyloriinfected individuals, and expression of DC-SIGN was determined by immunohistochemical staining. (A) Representative stainings are shown (original magnification, $\times 100 ; \times 400$ [inset]), (B) along with frequencies of DC-SIGN+ DCs, expressed as percentage of stained area in the gastric mucosa. Each symbol indicates an individual donor; horizontal lines represent medians. (C) Gastric DCs in lamina propria single cell preparations from uninfected and $\mathrm{H}$. pylori-infected patients undergoing gastrectomy were identified by flow cytometry as HLA-DR ${ }^{\text {hi }} \mathrm{DC}-\mathrm{SIGN}{ }^{+}$cells. Lower dot plots show staining with an isotype control for the DC-SIGN antibody. The percentages indicate cell frequencies among total live (7AAD-) cells. (D) Flow cytometric analysis of the immunophenotype of gastric HLA-DR hiDC-SIGN+ DCs. The solid line in histograms shows staining with the indicated antibody, and the dotted line shows staining with isotype controls. Data are shown for 3 uninfected and $3 \mathrm{H}$. pylori-infected individuals.

mutual exclusion of tolerogenic and immunogenic properties in a given DC population. To obtain functional evidence that DCs are required for the development and maintenance of neonatally acquired immunological tolerance to $H$. pylori infection, we infected mice that transgenically express the diphtheria toxin receptor under the control of the CD11c promoter (cd11c-DTR tg mice) at 1 week of age. At 6 weeks after infection, the $c d 11 c$-DTR tg mice as well as their nontransgenic, infected littermates and respective uninfected controls of both genotypes received 3 weekly doses of diphtheria toxin for 2 weeks (Figure 5F), resulting in the depletion of approximately $40 \%-50 \%$ of DCs in the gastric mucosa, MLNs, and spleen (Supplemental Figure 2 and data not shown). DC-depleted mice were colonized at significantly lower levels (Figure 5G) and exhibited higher levels of leukocyte and $\mathrm{CD}^{+} \mathrm{T}$ cell infiltration into the gastric mucosa than their DC-replete, infected littermates and uninfected controls (Figure 5, H and I). A higher proportion of $\mathrm{CD}^{+}$gastric mucosal $\mathrm{T}$ cells expressed IFN- $\gamma$ in the DC-depleted, infected mice than in the other 3 groups (Figure 5J). The infiltration of leukocytes into the gastric mucosa of DC-depleted, infected mice was histologically evident as mild to moderate gastritis, a feature that is never observed in neonatally infected, fully tolerant mice at such early time points after infection (Figure 5, K and L). In summary, the depletion of CD $11 \mathrm{c}^{+}$ DCs in the $c d 11 c$-DTR tg model reveals that the maintenance of H. pylori-specific immunological tolerance requires DCs, a finding that may be explained by the predominantly tolerogenic properties of H. pylori-experienced DCs in vitro and in vivo.

$D C$ infiltrating the gastric mucosa of $H$. pylori-infected patients express HLA-DR but lack costimulatory molecules. To examine the recruitment and localization of DCs to the gastric mucosa of H.pylori-infected individuals, we examined antral biopsies collected at endoscopy from $H$. pylori-infected and uninfected volunteers. Cryosections from antral biopsies were immunostained for DC-SIGN, a marker for human DCs (30). The large majority of DC-SIGN ${ }^{+}$DCs were 

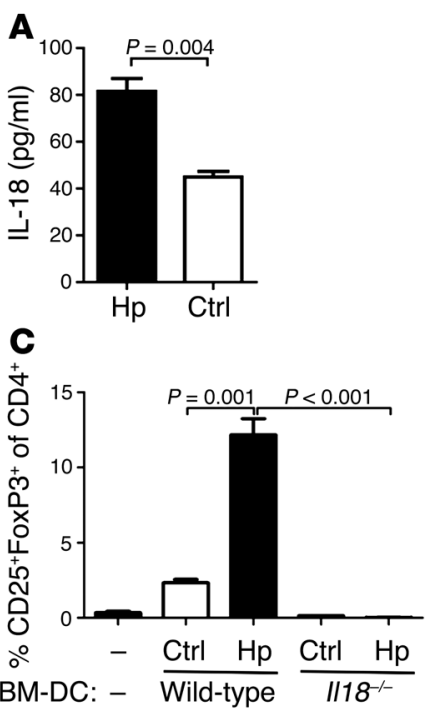

D

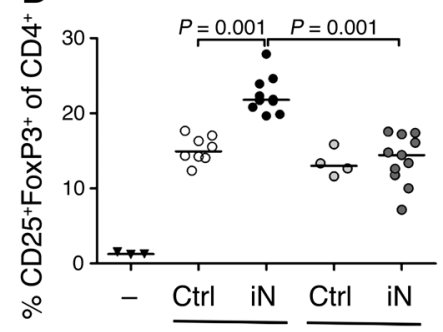

MLN DC: - Wild-type $\frac{}{1118^{-/}}$

F

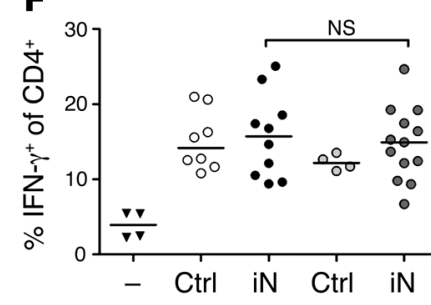

MLN DC: - $\overline{\text { Wild-type }} \frac{1118^{-1}}{19}$

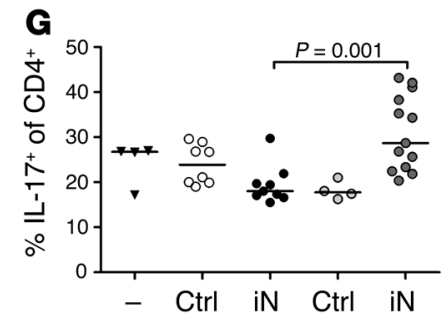

MLN DC: $-\overline{\text { Wild-type }} \frac{}{1 / 18^{-\alpha}}$
B
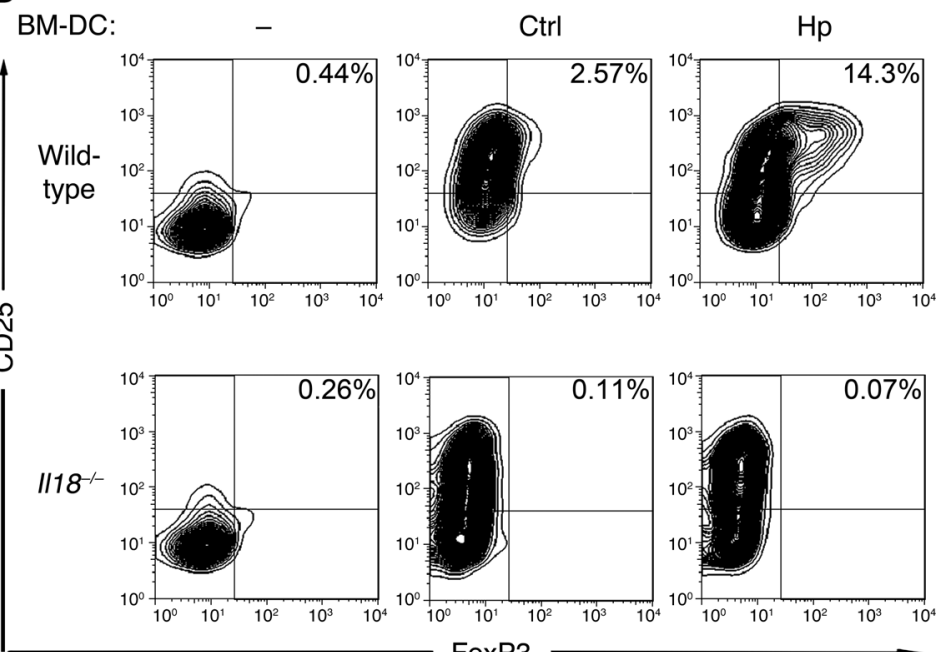

FoxP3

\section{$\mathbf{E}$}

MLN DC
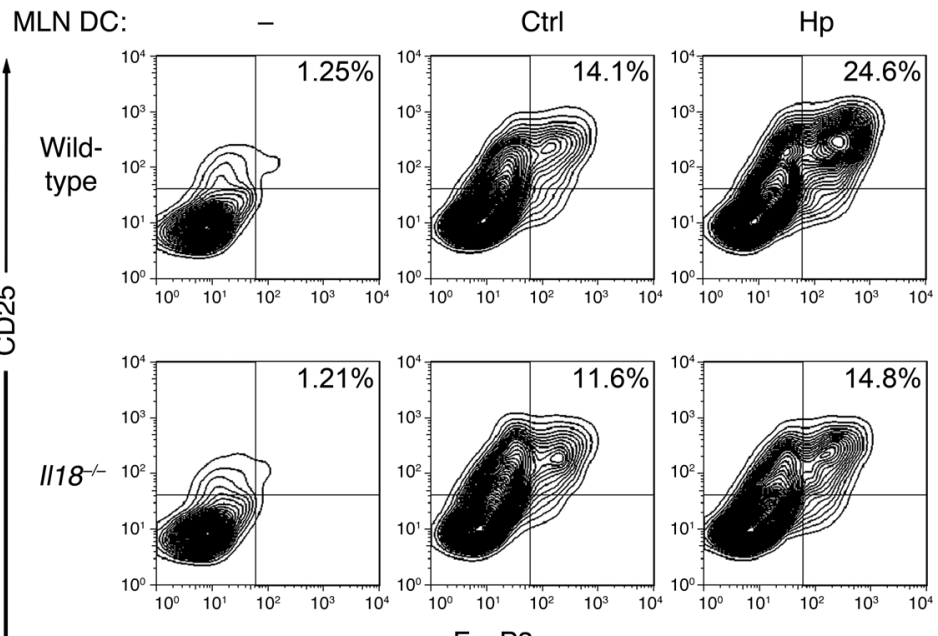

Figure 7

Treg induction by tolerogenic DCs requires IL-18. (A) Wild-type BM-DCs were infected with H. pylori PMSS1 (MOI 50), and IL-18 secretion was assessed by ELISA. (B and $\mathbf{C}$ ) Wild-type and $/ / 18^{-/-}$BM-DCs were infected as described in $\mathbf{A}$, and cocultured at a 1:2 ratio with immunomagnetically isolated, splenic OTII CD4+CD25- T cells for 3 days in the presence of rTGF- $\beta$, rlL-2, and anti-CD3\& mAb prior to the flow cytometric analysis of CD4, CD25, and FoxP3 expression. (B) Representative FACS plots of the CD4+ gate are shown, (C) along with mean \pm SEM of triplicate cocultures. (D-G) C57BL/6 and BL/6./118-- mice were infected at 7 days (iN) of age with 1 orogastric dose of $H$. pylori or remained uninfected. Upon sacrifice after 4 weeks after infection, CD11 $\mathrm{c}^{+} \mathrm{DC}$ were immunomagnetically isolated from single cell MLN suspensions of individual mice and cocultured for 3 days with splenic $C D 4^{+}$CD25- T cells at a 1:2 ratio in the presence of rIL-2 and anti-CD3ع $\mathrm{mAb}$ and $(\mathbf{D}$ and $\mathbf{E})$ with or $(\mathbf{F}$ and $\mathbf{G})$ without rTGF- $\beta$. (D and E) Cocultures were subjected to flow cytometric analysis of CD4, CD25, and FoxP3 expression (representative mice are shown in $\mathbf{E}$, and the quantification of all mice is shown in $\mathbf{D}$ ) or (F and $\mathbf{G})$ CD4 and IFN- $\gamma$ or IL-17 expression. Data shown are representative of at least 3 independent $(\mathbf{A}-\mathbf{C})$ in vitro and $(\mathbf{D}-\mathbf{G})$ in vivo experiments. Horizontal lines represent the median. (B and $\mathbf{E})$ Numbers indicate the percentage of $\mathrm{FOXP}^{+} \mathrm{CD} 25^{+}$cells. 
A
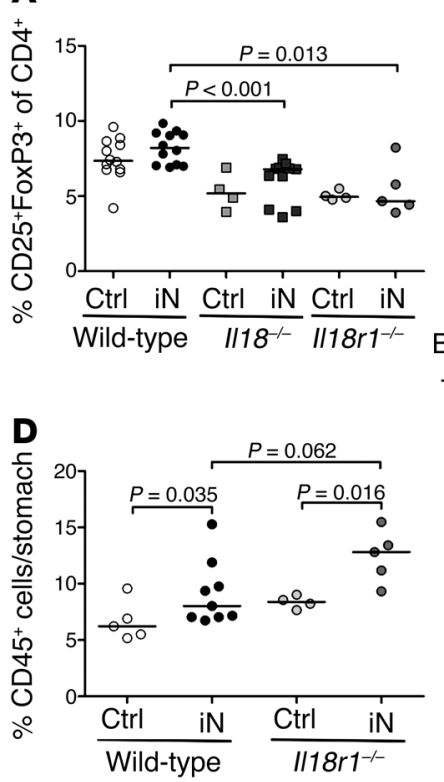
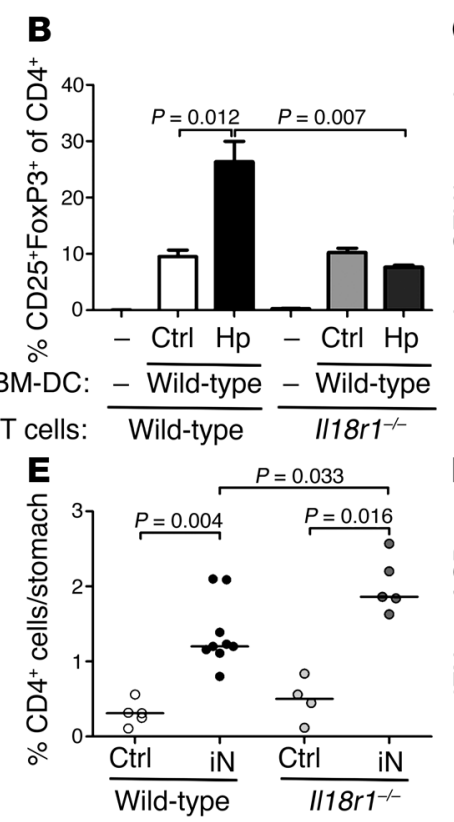

c
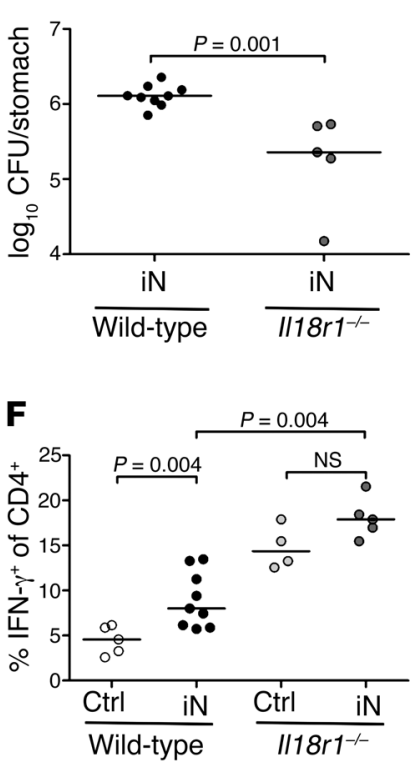

Figure 8

Treg differentiation and the development of $H$. pylori-specific tolerance requires IL-18 signaling in vivo. (A) Single cell MLN preparations from individual C57BL/6, BL/6. $/ 118^{-/-}$, and BL/6./118r1 $1^{-/-}$mice neonatally infected with $\mathrm{H}$. pylori were subjected to flow cytometric analysis of CD4, CD25, and FoxP3 expression and compared with respective uninfected controls. Each data point represents an individual mouse; horizontal lines indicate medians. (B) Wild-type BM-DCs were infected with $H$. pylori PMSS1 (MOI 50) and cocultured at a 1:2 ratio

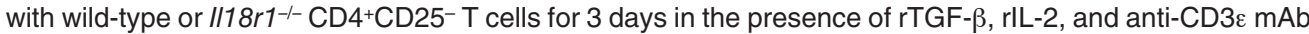
prior to analysis of CD4, CD25 and FoxP3 expression. Mean \pm SEM of triplicate cocultures are shown. (C-F) Wild-type C57BL/6 and BL/6./I18r1-l- mice were neonatally infected with $H$. pylori and sacrificed 4 weeks after infection. (C) Gastric H. pylori colonization. (D and E) Gastric mucosal CD45+ leukocyte and CD4+ T cell infiltration. (F) IFN- $\gamma$ expression by stomach-infiltrating $\mathrm{CD} 4^{+} \mathrm{T}$ cells, as determined by intracellular cytokine staining. Horizontal lines represent the median.

found scattered in the lamina propria of both uninfected and H. pylori-infected individuals (Figure 6A), and some DCs were positioned just underneath the epithelium. The frequency of DCSIGN ${ }^{+}$DCs was significantly higher in $H$. pylori-infected individuals compared with that in their uninfected counterparts (Figure 6B). To immunophenotypically characterize the DC-SIGN ${ }^{+}$DCs in the gastric mucosa of $H$. pylori-infected patients, we obtained gastric tissue from a set of 8 patients undergoing gastrectomy at Sahlgrenska University Hospital (of which 4 were $H$. pylori infected and 4 were uninfected). Lamina propria single cell suspensions were prepared, and DCs were identified by flow cytometry among live cells $\left(7 \mathrm{AAD}^{-}\right)$based on their expression of high levels of HLA-DR together with DC-SIGN (Figure 6C). The HLA-DR ${ }^{\text {hiDC-SIGN }}{ }^{+}$ DCs expressed the DC marker CD11c (data not shown) but no or only little CD80, CD83, or CD86 (Figure 6D). Furthermore, there were no consistent differences in DC phenotype when comparing H. pylori-infected and uninfected patients (Figure 6D). In summary, the results suggest that human gastric DCs, despite being actively recruited in large numbers to the $H$. pylori-infected mucosa, fail to upregulate activation markers and thus appear to retain a semimature phenotype. This observation may explain the ability of $H$. pylori to persist in the human gastric mucosa.

DC-intrinsic IL-18 expression is required for the conversion of naive T cells to Fox $3^{+}$Tregs in vitro. As part of our ongoing efforts to characterize the molecular players contributing to $H$. pylori-associated gastric
BM-DCs, independent of whether more efficiently than control CD3 cross-linking (Figure 7, B and C) or ovalbumin (Supplemental Figure 3, C and D). Interestingly, infected $I l 18^{-/-}$BM-DCs exhibited a clear defect in FoxP3 induction under both coculture conditions (Figure 7, B and C, and Supplemental Figure 3, C and D). In line with a reported role for IL-18 in Th1 differentiation in vitro, both uninfected and infected $I l 18^{-/-}$DCs failed to induce IFN- $\gamma$ expression in cocultured naive T cells (Supplemental Figure 4, A-D). We next sought to assess a possible role for mature IL-18 in generating tolerogenic DCs in the course of neonatal infection with $H$. pylori in vivo. As demonstrated above (Figure 5), immunomagnetically isolated MLN-DCs from neonatally infected wild-type mice were significantly better inducers of FoxP3 expression than MLN-DCs from naive mice (Figure 7, D and E). In contrast, MLN-DCs from infected Il18 ${ }^{-/}$mice did not induce FoxP3 expression in T cells any more efficiently than MLN-DCs from naive $I l 18^{-/-}$mice (Figure 7, D and E). Interestingly, whereas Treg differentiation induced by MLN-DCs was thus severely impaired due to the lack of DC-intrinsic IL-18 production (Figure 7, D and E), DCs from infected $I l 18^{-/-}$mice were equally good as wild-type DCs at inducing Th1 differentiation in this system (Figure 7F) and significantly better at inducing Th17 differentiation than wild-type DCs (Figure 7G). The combined results show that IL-18 production is induced in DCs upon infection with H. pylori and suggest a key role for DC-derived IL-18 in skewing T cell differentiation away from Th17 and toward Treg responses. 
A

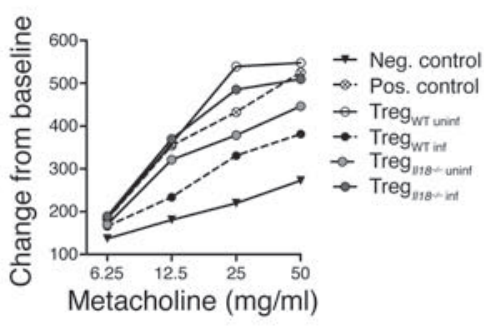

B

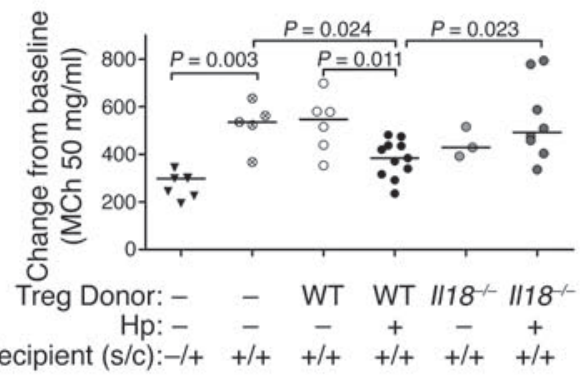

C

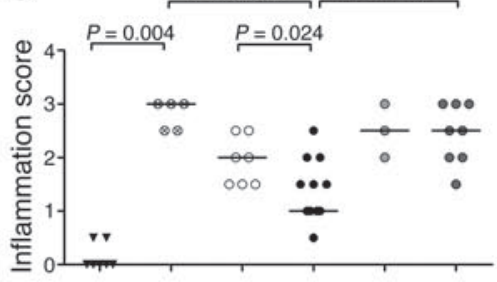

Treg Donor:- - WT WT $1118^{-} \| 18^{-}$

$\mathrm{Hp}:-\quad-\quad--\quad+\quad-\frac{+}{+}+$
D

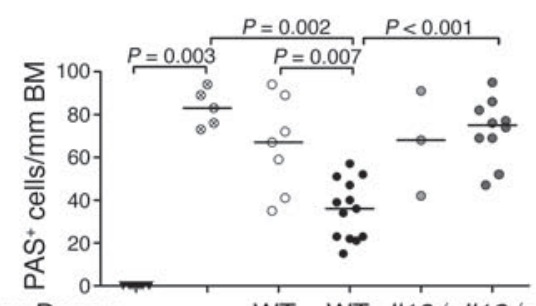

Treg Donor: - - WT WT $\left\|18^{\wedge}\right\| 18^{\prime}$

$\begin{array}{cccccc}\mathrm{Hp}:- & - & - & + & - & + \\ \mathrm{s} / \mathrm{c}):-/+ & +/+ & +/+ & +/+ & +/+ & +/+\end{array}$

Recipient (s/c): $-/+\quad+/+\quad+/+\quad+/+\quad+/+\quad+/+$

$\mathbf{F}$

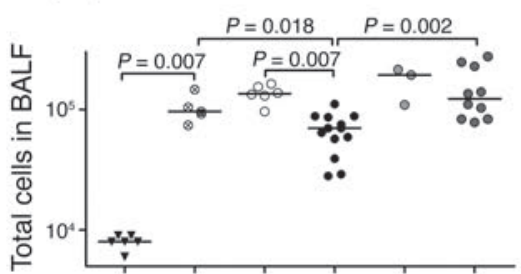

Treg Donor: - - WT WT $\| 18^{--118^{-}}$

$\begin{array}{cccccc}\text { Hp: }- & - & - & + & - & + \\ \text { Recipient (s/c): }-/+ & +/+ & +/+ & +/+ & +/+ & +/+\end{array}$

G

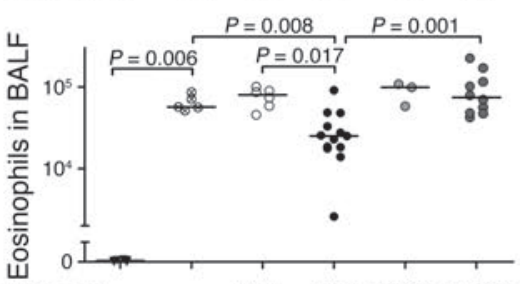

Treg Donor: - _ - WT WT $\left\|18^{-}\right\| 18^{-}$

Hp: - $\quad-\quad+-+$

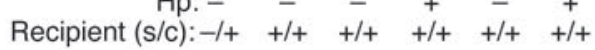

E
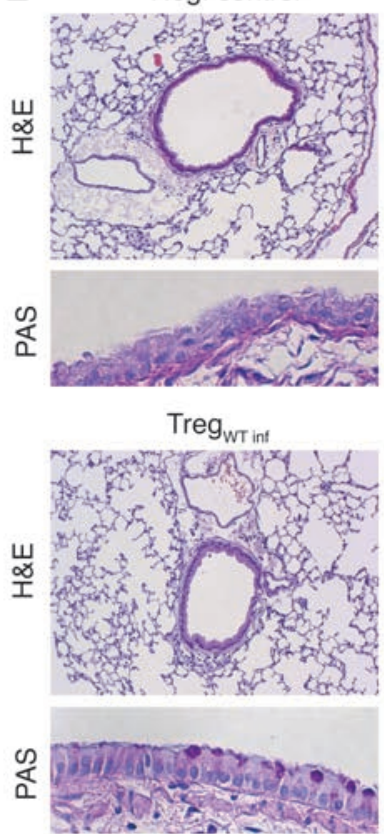
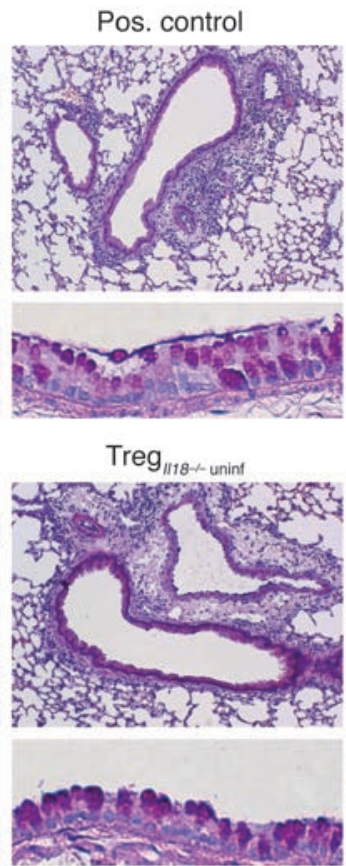

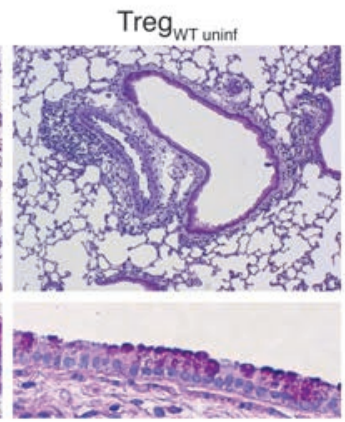

Treg $_{1118-\text { int }}$

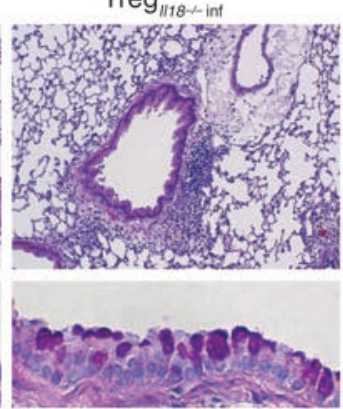

Figure 9

IL-18 signaling is required for the differentiation of functional Tregs in vivo. Wild-type C57BL/6 mice were sensitized with 2 i.p. doses of alumadjuvanted ovalbumin prior to challenge with aerosolized ovalbumin 2 weeks after the last sensitization. Four groups of sensitized recipients received 250,000 immunomagnetically isolated $C D 4{ }^{+} C D 25^{+} T$ cells isolated from the pooled MLNs of uninfected or neonatally infected wild-type C57BL/6 or BL/6./118 ${ }^{-/-}$donors (4-6 per group) 1 day before the first challenge. Negative controls were challenged without prior sensitization. (A and B) Airway hyperresponsiveness, (A) as assessed by challenge with increasing doses of methacholine and (B) the highest dose of $50 \mathrm{mg} / \mathrm{ml}$, respectively. (C-E) Tissue inflammation and goblet cell metaplasia, as assessed on H\&E- and PAS-stained tissue sections. Representative micrographs are shown in E. Original magnification, $\times 100$ (H\&E); $\times 400$ (PAS). Inflammation and PAS scores are shown in C and D, respectively. (F) Total cells contained in $1 \mathrm{ml} \mathrm{BALF.} \mathrm{(G)} \mathrm{Eosinophils} \mathrm{in} 1 \mathrm{ml} \mathrm{BALF}$. Horizontal lines indicate medians. s/c, sensitized/challenged.

IL-18 signaling is required for Treg differentiation and $H$. pylori-specific tolerance in vivo. Hypothesizing based on the above results that Treg numbers should consequently differ between the MLNs of wildtype and $\mathrm{Il} 18^{-/-}$mice, we quantified the proportion of FoxP3 ${ }^{+} \mathrm{CD} 25^{+}$ cells in the MLN CD4 $4^{+} \mathrm{T}$ cell population of neonatally infected wild-type and $I l 18^{-/-}$mice in comparison with that of uninfected controls of both genetic backgrounds. Whereas the infection induced an increase in MLN Tregs in wild-type mice, this was not observed in $I l 18^{-/-}$animals, resulting in significantly lower Treg numbers in infected $I l 18^{-/-}$animals compared with infected wildtype animals (Figure 8A). Interestingly, the same clear trend was observed in mice lacking the IL-18 receptor (IL-18R; Figure 8A), which also exhibited significantly lower Treg counts upon neonatal infection than wild-type mice. Hypothesizing that DC-derived 
IL-18 acts directly on $\mathrm{T}$ cells to facilitate their conversion to FoxP3 ${ }^{+} \mathrm{CD} 25^{+}$Tregs, we compared naive wild-type and $I l 18 r 1^{-/-} \mathrm{T}$ cells, with respect to their ability to upregulate FoxP3 upon coculture with $H$. pylori-experienced BM-DCs. Indeed, Il18r1-/- T cells showed a strongly reduced ability to upregulate FoxP3, indicating that IL-18 signaling in T cells is required for efficient Treg conversion in vitro (Figure $8 \mathrm{~B}$ ). We next examined the ability of Il18r $1^{-1-}$ mice to develop tolerance to $H$. pylori as a consequence of experimental infection during the neonatal period. Neonatally

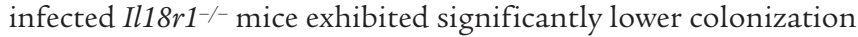
levels than wild-type mice infected at the same age (Figure 8C), which was accompanied by higher gastric leukocyte and $\mathrm{T}$ cell infiltration (Figure 8, D and E) and stronger gastric production of IFN- $\gamma$ and IL-17, as determined by intracellular cytokine staining and/or qPCR (Figure 8F and Supplemental Figure 5). We conclude from these results that T cell-intrinsic IL-18 signaling is essential for Treg differentiation in vitro and in vivo and is also required for the generation of Treg-mediated, neonatally acquired tolerance to $H$. pylori infection.

$\mathrm{CD} 4^{+} \mathrm{CD} 25^{+} \mathrm{T}$ cells isolated from neonatally infected wild-type mice but not Il18 $8^{-/}$or Il18r1 $1^{-/-}$mice suppress allergen-induced asthma. Given the requirement for DC-derived IL-18 in Treg differentiation in vitro and in vivo, we hypothesized that $\mathrm{CD} 4^{+} \mathrm{CD} 25^{+} \mathrm{T}$ cells immunomagnetically isolated from the MLNs of neonatally infected $I l 18^{-/-}$or $I l 18 \mathrm{rl}^{-/-}$mice should lack suppressive activity in a murine model of experimentally induced allergic airway disease. Having shown earlier that neonatal infection of wild-type mice with H. pylori generates Tregs with highly suppressive activity in the asthma model (23), we compared immunomagnetically isolated $\mathrm{CD} 4^{+} \mathrm{CD} 25^{+} \mathrm{T}$ cells from neonatally infected wild-type, $I l 18^{-/-}$, or Il18r1 $1^{-/-}$animals, with respect to their ability to inhibit experimentally induced asthma (Figure 9 and Supplemental Figure 6). Mice were sensitized i.p. with alum-adjuvanted ovalbumin and subsequently challenged by 3 consecutive daily exposures to aerosolized ovalbumin. Groups of mice were intravenously administered $\mathrm{CD} 4{ }^{+} \mathrm{CD} 25^{+} \mathrm{T}$ cells isolated from either neonatally infected wildtype, $I l 18^{-/-}$, or $I l 18 \mathrm{r}^{-/-}$animals or from age-matched uninfected controls of each genotype 1 day prior to the first challenge. All mice were examined with respect to clinical and histopathological parameters of asthma development 2 days after the last challenge. Sensitized, challenged mice exhibited increased airway resistance compared with nonsensitized controls, as assessed by methacholine challenge, which could be prevented by $\mathrm{CD} 4^{+} \mathrm{CD} 25^{+} \mathrm{T}$ cells from infected wild-type animals but not infected $\mathrm{Il1} \mathrm{8}^{-/-}$animals (Figure 9, A and B) or Il18r1-1- animals (Supplemental Figure 6, $\mathrm{A}$ and $\mathrm{B}) . \mathrm{CD}^{+} \mathrm{CD} 25^{+} \mathrm{T}$ cells from uninfected control groups did not prevent allergen-induced airway resistance (Figure 9, A and B). Similarly, the lung inflammation and $\mathrm{PAS}^{+}$goblet cell metaplasia that are hallmarks of asthma in this model were prevented by $\mathrm{CD} 4^{+} \mathrm{CD} 25^{+} \mathrm{T}$ cells from infected wild-type mice but not infected $I l 18^{-/-}, I l 18 r 1^{-/-}$, or uninfected mice (Figure 9, C-E, and Supplemental Figure 6, C and D). A quantitative analysis of allergen-induced bronchoalveolar inflammation and eosinophilia confirmed these findings (Figure 9, F and G, and Supplemental Figure 6, E and F). Interestingly, quantitative RT-PCR analysis of IL-10 expression performed on freshly purified $\mathrm{CD} 4^{+} \mathrm{CD} 25^{+} \mathrm{T}$ cells revealed that $\mathrm{T}$ cells isolated from infected wild-type mice, but not infected Il18 ${ }^{-/-}$mice, expressed copious amounts of IL-10 compared with $\mathrm{CD} 4{ }^{+} \mathrm{CD} 25^{+} \mathrm{T}$ cells from the corresponding uninfected donors (Supplemental Figure 6, G and H), implying that this cytokine may be key in asthma protection by H. pylori-induced Tregs. Overall, the results suggest that Treg differentiation and function is severely impaired under conditions of IL-18 or IL-18R deficiency, underscoring the importance of this cytokine in Treg biology.

\section{Discussion}

H. pylori infection is acquired during early childhood and typically persists for life (31). Chronic infection with $H$. pylori causes gastritis and predisposes to gastric cancer but has also been associated epidemiologically with protection against asthma (19, 20, 22), multiple sclerosis (32), and inflammatory bowel disease (33). The beneficial effects of the infection are particularly evident in the pediatric population, with respect to both asthma and colitis incidence $(21,34)$. Experimental infection with $H$. pylori during the neonatal period recapitulates the protective effects against asthma in mouse models of the disease (23). We show here that the exposure of DCs to $H$. pylori had profound effects on the properties of these cells in vitro and in vivo. H. pylori-experienced BM-DCs or MLN-DCs lose their ability to mature into fully immunogenic, stimulatory antigen-presenting cells and to activate effector functions in naive T cells. Instead, H. pylori exposure triggers tolerogenic properties in DCs, which become efficient inducers of Tregs in vitro and suppress $H$. pylori clearance and the associated immunopathology in vivo. Our results explain why the systemic depletion of DCs improves the immunological control of $H$. pylori in naive mice and enhances vaccine-induced protective immunity in immunized mice (12). Our BM-DC results suggest that direct contact between the bacteria and DCs is required for the observed effects, whereas a functional Cag pathogenicity island-encoded type IV secretion system is dispensable. Semimature DCs with high expression of HLA-DR but low expression of CD80, CD83, and CD86 constitute the predominant DC population in the stomachs of chronically infected humans; this finding is particularly intriguing, as DCs are actively recruited to the infected gastric mucosa but obviously fail to mature and, presumably, to acquire immunogenic/stimulatory properties. Our findings are reminiscent of a recent study by Robinson et al., who found that patients with peptic ulcer disease exhibited stronger Th1 and Th2 responses to H.pylori than asymptomatic carriers, whereas the latter group predominantly mounted Treg responses to the infection (35). IL-10-expressing Tregs were particularly abundant in the gastric mucosa of the asymptomatic carriers compared with patients with peptic ulcer disease; interestingly, mucosal IL-10 levels were directly correlated with bacterial densities, with asymptomatic carriers showing high IL-10 expression and receiving the highest colonization scores and patients with peptic ulcer disease showing low IL-10 expression and receiving comparatively low colonization scores (35). This interesting association argues that strong gastric Treg responses (perhaps induced by tolerogenic DCs) ensure persistent infection on the one hand and prevent severe immunopathology on the other.

Several previous reports have highlighted the fact that $H$. pylori possesses few, if any, PAMPs that might activate innate immune responses by binding to surface exposed, endosomal, or cytoplasmic pattern recognition receptors $(27,36,37)$. H. pylori flagellin is a poor ligand of TLR5 (36), and the bacterium's LPS consists predominantly of the biologically inactive tetra-acylated lipid A variety (38). Whereas TLR2 ligands clearly exist in $H$. pylori and related Helicobacter species $(26,27)$, the net effects of TLR2 ligation are antiinflammatory rather than proinflammatory (27). We show here that the DC-derived cytokine IL-18 plays a crucial role in the 
tolerogenic activity of $H$. pylori-experienced DCs in vitro and in vivo. In contrast to wild-type cells, BM-DCs derived from $I l 18^{-/-}$ mice failed to induce FoxP3 expression in naive T cells upon exposure to $H$. pylori; more importantly, $I l 18^{-/-}$MLN-DCs from neonatally infected mice exhibited the same defect in Treg induction relative to their wild-type counterparts. DC-derived IL-18 appears to act directly on $\mathrm{T}$ cells and to promote their conversion to Tregs: IL-18R-deficient T cells failed to upregulate FoxP3 upon coculture with tolerogenic DCs, and both IL-18- and IL-18R-deficient mice exhibited strongly decreased overall Treg numbers in the draining MLNs. Furthermore, $\mathrm{CD}^{+} \mathrm{CD} 25^{+} \mathrm{MLN}$ T cells isolated from both gene-targeted strains are less capable of suppressing allergic airway disease in an allergen-induced sensitization and challenge model. All available data thus suggest that IL-18 contributes to H. pylori persistence and fine-tunes the balance between infection control on the one hand and excessive immunopathological $\mathrm{T}$ cell responses on the other. The results are confirmed in vivo by our observation that $I l 18 \mathrm{rI}^{-/-}$mice failed to develop immune tolerance to neonatal infection with $H$. pylori. Our data are in line with 2 recent studies demonstrating a protective role for IL-18, produced upon activation of the NLRP3 inflammasome, in DSS-induced colitis $(39,40)$. In this model, lack of expression of NLRP3, caspase-1, and ASC in intestinal epithelial cells rendered mice hypersusceptible to DSS-induced colitis $(39,40)$.

As mentioned earlier, the protection against asthma is mediated by $H$. pylori-induced Tregs in experimentally infected mice: asthma protection is abrogated by the systemic depletion of Tregs and can be adoptively transferred from infected donors to uninfected recipients via Tregs (23). An interesting characteristic of neonatally infected mice that have been subjected to an asthma-inducing protocol is their pulmonary infiltration by highly suppressive Tregs and by semimature DCs (23). Our finding that $H$. pylori-exposed DCs are incapable of sensitizing mice to ovalbumin when administered intranasally makes it seem likely that $H$. pylori-mediated asthma protection is a direct consequence of the reprogramming of DCs in vivo. However, as DCs are not known to migrate between the mucosal surfaces of the lung and gastrointestinal tract, it appears likely that highly mobile Tregs are induced in the MLNs and then migrate to the airways, in which they exert their suppressive activity and protect against allergen-induced asthma. DCs and Tregs efficiently regulate one another in a negative feedback loop, promoting a tolerogenic, immunosuppressive environment. For instance, Onishi et al. reported that FoxP3 ${ }^{+}$Tregs form aggregates on DCs, thereby actively downregulating their costimulatory molecules and maturation markers and impairing the ability of DCs to activate antigen-specific, naive $\mathrm{T}$ cells (41). In summary, we show here that $H$. pylori possesses the distinct ability to reprogram DCs toward a tolerogenic phenotype in vitro and in vivo, a process that ensures persistence of the bacteria in the host and may cross-protect against chronic inflammatory and autoimmune diseases. The semimature phenotype of lamina propria DCs that is a hallmark of the gastric mucosa of asymptomatic human $H$. pylori carriers likely represents the human cellular counterpart of the murine tolerogenic DC population.

\section{Methods}

Animal experimentation; bronchoalveolar lavage, lung, and gastric histopathology; and assessment of H. pylori colonization. C57BL/ 6 wild-type, BL/6.Tlr2--, $\mathrm{BL} / 6 . \mathrm{My} d 88^{-/}$, OT II TCR transgenic, and $c d 11 c$-DTR tg mice were originally purchased from Charles River Laboratories; BL/6.Il18--, BL/6.Ill8r1 $1^{-/}$, and
cd11c-hDC-SIGN transgenic mice were obtained from Wolf-Dietrich Hardt (Federal Institute of Technology, Zürich, Switzerland), Burkhard Becher (University of Zürich), and Tim Sparwasser (Medical School, University of Hannover, Hannover, Germany). All mice were bred at a University of Zurich specific pathogen-free facility. Mice were housed in individually ventilated cages. Mixed gender groups were infected at either 7 days or 6 weeks of age with 1 orogastric dose of approximately $2 \times 10^{7} \mathrm{CFUs} \mathrm{H}$. pylori PMSS1 (18). In vivo depletion of DCs was achieved by i.p. injections of $4 \mathrm{ng}$ diphtheria toxin per g of body weight at 2-day intervals for 2 weeks. For asthma induction, mice were sensitized either by 2 i.p. injections of $20 \mu \mathrm{g}$ ovalbumin (Sigma-Aldrich) emulsified in $2.25 \mathrm{mg}$ aluminum hydroxide (Alum Imject; Pierce) with a 2 -week interval or by intranasal delivery of $1 \times 10^{6} \mathrm{BM}$-DCs loaded overnight (o/n) with $20 \mu \mathrm{g} / \mathrm{ml}$ ovalbumin (Sigma-Aldrich). Irrespective of the mode of sensitization, mice were challenged with $1 \%$ aerosolized ovalbumin using an ultrasonic nebulizer (NE-U17; Omron) for 20 minutes daily on days 14,15 , and 16 after (second) sensitization. Airway resistance measurements were performed on anesthetized, intubated, and mechanically ventilated mice (FlexiVent, Scireq) in response to increasing doses of inhaled methacholine, as described previously (42). Lungs were lavaged via the trachea with $1 \mathrm{ml}$ PBS. BALF cells were counted using trypan blue dye exclusion. Differential cell counts of lymphocytes, neutrophils, and eosinophils were performed on cytocentrifuged preparations stained with the Microscopy Hemacolor Set (Merck). Lungs were fixed by inflation and immersion in $10 \%$ formalin and embedded in paraffin. Lung tissue sections were stained with H\&E and PAS and were examined on a BX40 Olympus microscope in blinded fashion by 2 independent experimenters. Peribronchial inflammation was scored on a scale from 0 to 4 on 5 randomly chosen areas per slide, as described previously (43). PAS-positive goblet cells were quantified per $1 \mathrm{~mm}$ of basement membrane in the primary bronchus and several medium-sized bronchi using Soft Imaging Systems software. Stomachs were retrieved and dissected longitudinally into equally sized pieces. For the quantitative assessment of $H$. pylori colonization, 1 stomach section was homogenized in Brucella broth, and serial dilutions were plated on horse blood plates for colony counting as described previously (18). For the quantitative assessment of gastric histopathology, Giemsa-stained paraffin-embedded stomach sections were scored on a scale from 0 to 6 for the parameters of chronic inflammation, atrophy, epithelial hyperplasia, and metaplasia, as described in detail previously (10). All gastric histopathology images were taken at $\times 100$ or $\times 200$ final magnification on a Leica Leitz DM $\mathrm{RB}$ microscope equipped with a DFC 420C camera. Images were acquired using Leica Application Suite 3.3.0 software.

Volunteers and assessment of gastritis and H. pylori colonization. Volunteers were recruited among blood donors at Sahlgrenska University Hospital after serologic analysis, and $H$. pylori infection was subsequently confirmed or excluded by culture on Scirrow plates. Gastric antrum biopsies were collected from $8 \mathrm{H}$. pylori-infected volunteers ( 7 males and 1 female, aged between $25-57$ years) and 10 uninfected volunteers (5 males and 5 females, aged between 23-62 years) by endoscopy, immediately frozen in OCT medium, and later used for immunohistochemical staining of DCs. In addition, 1 biopsy from each volunteer was fixed in formalin, paraffin embedded, and examined by an experienced histopathologist for the grade of gastritis and the presence of Helicobacter-like organisms (HLOs) using the updated Sydney system (44). The serology, culture, and pathology results agreed in all cases, except those for 1 individual who did not have visible HLOs in the pathological examination but was culture and serology positive. Biopsy samples from 6 uninfected subjects were histologically normal without inflammation or HLOs, and biopsy samples from 4 uninfected subjects had mild chronic gastritis (score of 1) but no HLOs or active inflammation. In contrast, active chronic inflammation and HLOs were observed in biopsy samples from antrum of all $H$. pylori-infected subjects. The H. pylori- 
infected individuals had a chronic inflammation score of $1.9 \pm 0.4$ (mean \pm standard deviation), an active inflammation score of $1.4 \pm 0.7$, and an HLO score of $1.9 \pm 0.8$. Atrophy and metaplasia was seen in 1 of the $H$. pylori-infected subjects, and metaplasia alone was seen in 1 of the H. pylori-infected subjects.

Immunohistochemical and flow cytometric analysis of human gastric DCs. Antrum biopsies from $H$. pylori-infected and uninfected volunteers were cryosectioned into $8-\mu \mathrm{m}$ thick sections, fixed for 10 minutes in ice-cold acetone, and endogenous peroxidase blocked with glucose-oxidase for 20 minutes. Sections were stained with mouse monoclonal antibodies to DC-SIGN (clone DCN46, BD Biosciences) in PBS containing 5\% human and rabbit serum at room temperature for 30 minutes, followed by an HRP-conjugated rabbit antibody to mouse immunoglobulins (DakoCytomation). Isotype control antibodies were always run in parallel. The area stained with the respective antibodies was calculated relative to the total area of each tissue section, using Biopix image analysis software. For isolation of human gastric cells, $4 \mathrm{H}$. pylori-infected patients $(2$ males and 2 females, aged between 64-81 years) and 4 uninfected patients ( 1 male and 3 females, aged between 61-75 years) undergoing surgery for gastric adenocarcinoma $(n=2)$, pancreas cancer $(n=5)$, or chronic pancreatitis $(n=1)$ at Sahlgrenska University Hospital were also included in the study. Tissue was collected from antrum, and, in the gastric cancer patients, tissue was removed at least $5 \mathrm{~cm}$ away from the tumor. None of the patients had undergone radiotherapy or chemotherapy prior to the operation. $H$. pylori infection was determined based on serology, culture, and pathology reports; culture and serology agreed in 6 out of the 8 cases. Two seropositive cases with negative culture results, presumably due to extensive gastric atrophy and metaplasia, were deemed H. pylori positive. Antral tissue was stripped of muscle and connective tissue and cut into $5-\mathrm{mm}$ pieces. Epithelial cells were removed by incubating the tissue for $15 \mathrm{~min}$ utes at $37^{\circ} \mathrm{C}$ with $2 \mathrm{mM}$ EDTA and $1 \mathrm{mM}$ dithiothreitol (Sigma-Aldrich) in Hank's balances salt solution supplemented with $2 \%$ FCS for a total of 3 times. Lamina propria leukocytes were then released by incubating the remaining tissue for 1 hour at $37^{\circ} \mathrm{C}$ with $100 \mathrm{U} / \mathrm{ml}$ collagenase type VIII (Sigma-Aldrich) and $0.1 \mathrm{mg} / \mathrm{ml}$ DNAse (Sigma-Aldrich) in RPMI 1640 containing $10 \%$ FCS. Isolated cells were stained with anti-CD80 (clone L307.4), CD86 (2331), CD83 (HB15e), DC-SIGN (DCN46), and HLA-DR (L243; all from BD Biosciences), and appropriate isotype controls were used. 7-Aminoactinomycin D (7AAD, Sigma-Aldrich) was used to exclude dead cells. Samples were acquired on an LSR-II flow cytometer (BD Biosciences) and analyzed using FlowJo software.

Preparation of murine BM-DCs and MLN-DCs and DC/T cell cocultures. For generation of BM-DCs, bone marrow isolated from the hind legs of wildtype, Tlr $2^{-/-}, M y d 88^{-/-}$, or $c d 11 c$-hDC-SIGN transgenic donor mice was seeded at 50,000 cells per well in 96-well plates in RPMI/10\% FCS and $4 \mathrm{ng} / \mathrm{ml}$ GM-CSF and cultured for 7 days. E. coli LPS (Serotype 0111:B4, SigmaAldrich) and the synthetic lipopeptide Pam3Cys-SKKKK (Genaxxon) were added at $0.5 \mu \mathrm{g} / \mathrm{ml}$ and $5 \mu \mathrm{g} / \mathrm{ml}$ final concentration, respectively, to induce BM-DC maturation. For the isolation of MLN-DCs, MLNs of individual mice were digested in $1 \mathrm{mg} / \mathrm{ml}$ collagenase (Sigma-Aldrich) for 30 minutes at $37^{\circ} \mathrm{C}$, with shaking prior to filtering through a cell strainer $(40 \mu \mathrm{m}$; BD Biosciences) and immunomagnetic isolation of DCs using mouse-specific CD11c microbeads (Miltenyi Biotec). DC cultures were infected o/n with wild-type H. pylori PMSS1 or an isogenic mutant lacking the cagE gene ( $\triangle$ CagE) described previously (18). Bacteria were killed with $200 \mathrm{U}$ penicillin/ $0.2 \mathrm{mg}$ streptomycin $/ \mathrm{ml}$ for 6 hours prior to the addition of T cells.
CD4 ${ }^{+} \mathrm{CD} 25^{-} \mathrm{T}$ cells were prepared from single cell suspensions of naive C57BL/6 spleens by immunomagnetic sorting (R\&D Systems). DCs were cocultured with $\mathrm{CD}^{+} \mathrm{CD} 25^{-} \mathrm{T}$ cells at a ratio of $1: 2\left(0.5 \times 10^{5} \mathrm{DCs}\right.$ to $1 \times 10^{5}$ T cells) in RPMI containing $10 \%$ FCS, $10 \mathrm{ng} / \mathrm{ml}$ recombinant TGF- $\beta$ (rTGF- $\beta$ ) (PeproTech), $10 \mathrm{ng} / \mathrm{ml}$ recombinant IL-2 (rIL-2) (R\&D Systems), and $1 \mu \mathrm{g} / \mathrm{ml}$ anti-CD $3 \varepsilon$ (BD Bioscience). After 72 hours of coculture, the cells were stained first for CD4 and CD25 and then, after fixation and permeabilization, for FoxP3 (FoxP3-APC, eBioscience). The percentage of FoxP3 ${ }^{+}$ $\mathrm{CD}^{+} \mathrm{T}$ cells was assessed by FACS. IFN- $\gamma$ secreted by cocultured DCs and $\mathrm{T}$ cells was quantified by ELISA (R\&D Systems). IL12p40 and IL-10 (both from BD Biosciences) and IL-18 (MBL) production by DCs was assessed by ELISA. The quantification of IL-10 production by RT-PCR is described in the Supplemental Methods.

Preparation of murine gastric single cell suspensions and flow cytometry. Onesixth of every stomach (antrum and corpus) and corresponding MLNs were digested in $1 \mathrm{mg} / \mathrm{ml}$ collagenase for 30 minutes at $37^{\circ} \mathrm{C}$, with shaking prior to mechanical disruption between glass slides and filtering through a cell strainer $(40 \mu \mathrm{m})$. Single cell suspensions were stained directly for FACS analysis. The following antibodies were used: CD11c-biotin, CD4-FITC, CD4-APC, CD86-FITC, and CD40-APC (all from BD Biosciences) as well as CD45-PB and CD80-APC (both from BioLegend). IFN- $\gamma$-PE-Cy7 (BD) and FoxP3-APC (eBioscience) were used for intracellular staining. Prior to intracellular cytokine staining, cells were stimulated and blocked in medium containing $2.5 \mu \mathrm{g} / \mathrm{ml}$ Brefeldin A (AppliChem), 0.2 $\mu \mathrm{M}$ ionomycin (Santa Cruz Biotechnology Inc.), and $50 \mathrm{ng} / \mathrm{ml}$ phorbol 12-myristate 13-acetate (Sigma-Aldrich) for 5 hours; stained for extracellular markers; and fixed in 4\% paraformaldehyde. Flow cytometry was performed on a Cyan ADP 9 instrument (Beckman Coulter) and analyzed using FlowJo software (TreeStar).

Statistics. GraphPad Prism (GraphPad Software) was used for statistical analyses. All $P$ values were calculated by Mann-Whitney test unless otherwise indicated. In column bar graphs, the SEM is indicated by vertical bars. $P$ values of less than 0.05 were considered statistically significant.

Study approval. All human studies were approved by the Regional Research Ethics Committee of Western Sweden, and informed consent was obtained from all participants. All animal experimentation was reviewed and approved by the Zurich Cantonal veterinary office $(63 / 2008$ and $170 / 2009$ to A. Müller).

\section{Acknowledgments}

We thank all volunteers and patients for their participation in the study. This study was supported by the Swiss National Science Foundation and the Zurich University Research Priority Program in Systems Biology (to A. Müller), the Deutsche Forschungsgemeinschaft and Research Center for Immunology of the University of Mainz (to C. Taube), and the Swedish Science Council and Foundation for Strategic Research through the MIVAC centre of excellence (to M. Quiding-Järbrink). We are grateful to Burkhard Becher, WolfDietrich Hardt, and Tim Sparwasser for providing mouse strains.

Received for publication September 15, 2011, and accepted in revised form December 14, 2011.

Address correspondence to: Anne Müller, Institute of Molecular Cancer Research, University of Zürich, Winterthurerstr. 190, 8057 Zürich, Switzerland. Phone: 41.44.635.3474; Fax: 41.44.635.3484; E-mail: mueller@imcr.uzh.ch.
1. Maldonado RA, von Andrian UH. How tolerogenic dendritic cells induce regulatory $\mathrm{T}$ cells. Adv Immunol. 2010;108:111-165.

2. Banchereau J, et al. Immunobiology of dendritic against bacterial pathogens. Semin Immunol. 2009;
21(4):199-207.

4. Ishii KJ, Koyama S, Nakagawa A, Coban C, Akira S. Host innate immune receptors and beyond: making sense of microbial infections. Cell Host Microbe. 
2008;3(6):352-363

5. Kretschmer K, Apostolou I, Hawiger D, Khazaie K, Nussenzweig MC, von Boehmer H. Inducing and expanding regulatory $\mathrm{T}$ cell populations by foreign antigen. Nat Immunol. 2005;6(12):1219-1227.

6. Marshall BJ, Warren JR. Unidentified curved bacilli in the stomach of patients with gastritis and peptic ulceration. Lancet. 1984;1(8390):1311-1315.

7. Parsonnet J, et al. Helicobacter pylori infection and the risk of gastric carcinoma. $N$ Engl J Med. 1991; 325(16):1127-1131.

8. Parsonnet J, et al. Helicobacter pylori infection and gastric lymphoma. N Engl J Med. 1994; 330(18):1267-1271.

9. Pritchard DM, Crabtree JE. Helicobacter pylori and gastric cancer. Curr Opin Gastroenterol. 2006; 22(6):620-625

10. Sayi A, et al. The CD4+ T cell-mediated IFN-gamma response to Helicobacter infection is essential for clearance and determines gastric cancer risk. J Immunol. 2009;182(11):7085-7101.

11. Akhiani AA, et al. Protection against Helicobacter pylori infection following immunization is IL-12 dependent and mediated by Th1 cells. J Immunol. 2002;169(12):6977-6984.

12. Hitzler I, Oertli M, Becher B, Agger EM, Müller A. Dendritic cells prevent rather than promote immunity conferred by a helicobacter vaccine using a mycobacterial adjuvant. Gastroenterology. 2011 141(1):186-196.

13. Fox JG, Wang TC. Inflammation, atrophy, and gastric cancer. J Clin Invest. 2007;117(1):60-69.

14. Rieder G, Merchant JL, Haas R. Helicobacter pylori cag-type IV secretion system facilitates corpus colonization to induce precancerous conditions in Mongolian gerbils. Gastroenterology. 2005; 128(5):1229-1242.

15. Correa P. A human model of gastric carcinogenesis. Cancer Res. 1988;48(13):3554-3560.

16. Toller IM, Altmeyer M, Kohler E, Hottiger MO, Muller A. Inhibition of ADP ribosylation prevents and cures helicobacter-induced gastric preneoplasia. Cancer Res. 2010;70(14):5912-5922.

17. Toller IM, Hitzler I, Sayi A, Mueller A. Prostaglandin E2 prevents Helicobacter-induced gastric preneoplasia and facilitates persistent infection in a mouse model. Gastroenterology. 2010;138(4):1455-1467.

18. Arnold IC, et al. Tolerance rather than immunity protects from Helicobacter pylori-induced gastric preneoplasia. Gastroenterology. 2011;140(1):199-209.

19. Blaser MJ, Chen Y, Reibman J. Does Helicobacter pylori protect against asthma and allergy? Gut. 2008; 57(5):561-567.

20. Chen Y, Blaser MJ. Inverse associations of Helicobacter pylori with asthma and allergy. Arch Intern Med. 2007;167(8):821-827.

21. Chen Y, Blaser MJ. Helicobacter pylori colonization is inversely associated with childhood asthma. J Infect Dis. 2008;198(4):553-560.

22. Reibman J, et al. Asthma is inversely associated with Helicobacter pylori status in an urban population. PLoS One. 2008;3(12):e4060.

23. Arnold IC, et al. Helicobacter pylori infection prevents allergic asthma in mouse models through the induction of regulatory T cells. J Clin Invest. 2011; 121(8):3088-3093.

24. Gringhuis SI, den Dunnen J, Litjens M, van der Vlist M, Geijtenbeek TB. Carbohydrate-specific signaling through the DC-SIGN signalosome tailors immunity to Mycobacterium tuberculosis, HIV-1 and Helicobacter pylori. Nat Immunol. 2009; 10(10):1081-1088

25. Schaefer M, et al. Decreased pathology and prolonged survival of human DC-SIGN transgenic mice during mycobacterial infection. I Immunol. 2008; 180(10):6836-6845.

26. Rad R, et al. Extracellular and intracellular pattern recognition receptors cooperate in the recognition of Helicobacter pylori. Gastroenterology. 2009, 136(7):2247-2257.

27. Sayi A, et al. TLR-2-activated B cells suppress Helicobacter-induced preneoplastic gastric immunopathology by inducing $\mathrm{T}$ regulatory-1 cells. J Immunol. 2011;186(2):878-890.

28. Kiriya K, et al. Essential role of Peyer's patches in the development of Helicobacter-induced gastritis. Int Immunol. 2007;19(4):435-446.

29. Nagai S, et al. Role of Peyer's patches in the induction of Helicobacter pylori-induced gastritis. Proc Natl Acad Sci US A. 2007·104(21):8971-8976.

30. Geijtenbeek TB, et al. Identification of DC-SIGN, a novel dendritic cell-specific ICAM-3 receptor that supports primary immune responses. Cell. 2000; 100(5):575-585.

31. Weyermann M, Rothenbacher D, Brenner H. Acqui sition of Helicobacter pylori infection in early childhood: independent contributions of infected mothers, fathers, and siblings. Am J Gastroenterol. 2009; 104(1):182-189.

32. $\mathrm{Li} \mathrm{W}$, et al. Helicobacter pylori infection is a potential protective factor against conventional multiple sclerosis in the Japanese population. J Neuroimmunol.
2007;184(1-2):227-231.

33. Luther J, Dave M, Higgins PD, Kao JY. Association between Helicobacter pylori infection and inflammatory bowel disease: a meta-analysis and systematic review of the literature. Inflamm Bowel Dis. 2010;16(6):1077-1084.

34. Sladek M, Jedynak-Wasowicz U, Wedrychowicz A Kowalska-Duplaga K, Pieczarkowski S, Fyderek $\mathrm{K}$. [The low prevalence of Helicobacter pylori gastritis in newly diagnosed inflammatory bowel disease children and adolescent]. Przegl Lek. 2007; 64 suppl 3:65-67.

35. Robinson K, et al. Helicobacter pylori-induced peptic ulcer disease is associated with inadequate regulatory T cell responses. Gut. 2008;57(10):1375-1385.

36. Gewirtz AT, Yu Y, Krishna US, Israel DA, Lyons SL, Peek RM Jr. Helicobacter pylori flagellin evades toll-like receptor 5-mediated innate immunity. J Infect Dis. 2004;189(10):1914-1920.

37. Moran AP. Structure and conserved characteristics of Campylobacter jejuni lipopolysaccharides. J Infect Dis. 1997;176 suppl 2:S115-S121.

38. Moran AP, Lindner B, Walsh EJ. Structural characterization of the lipid A component of Helicobacter pylori rough- and smooth-form lipopolysaccharides. J Bacteriol. 1997;179(20):6453-6463.

39. Zaki MH, Boyd KL, Vogel P, Kastan MB, Lamkanfi M, Kanneganti T-D. The NLRP3 inflammasome protects against loss of epithelial integrity and mortality during experimental colitis. Immunity. 2010;32(3):379-391.

40. Dupaul-Chicoine J, et al. Control of intestinal homeostasis, colitis, and colitis-associated colorectal cancer by the inflammatory caspases. Immunity. 2010;32(3):367-378

41. Onishi Y, Fehervari Z, Yamaguchi T, Sakaguchi S. Foxp3+ natural regulatory $\mathrm{T}$ cells preferentially form aggregates on dendritic cells in vitro and actively inhibit their maturation. Proc Natl Acad Sci US A. 2008;105(29):10113-10118.

42. Reuter $S$, et al. Mast cell-derived tumour necrosis factor is essential for allergic airway disease. Eur Respir J. 2008;31(4):773-782.

43. Bopp $\mathrm{T}$, et al. Inhibition of cAMP degradation improves regulatory $\mathrm{T}$ cell-mediated suppression. J Immunol. 2009;182(7):4017-4024.

44. Dixon MF, Genta RM, Yardley JH, Correa P. Classification and grading of gastritis. The updated Sydney System. International Workshop on the Histopathology of Gastritis, Houston 1994. Am J Surg Pathol. 1996;20(10):1161-1181. 